\title{
ИДЕНТИФИКАЦИЯ ПАРАМЕТРОВ МЕХАНИЧЕСКОЙ СИСТЕМЫ ВИБРАЦИОННОГО ЭЛЕКТРОМАГНИТНОГО АКТИВАТОРА ПО ГРАНИЧНЫМ ОКОЛОРЕЗОНАНСНЫМ ЧАСТОТАМ
}

Гаврилин Алексей Николаевич', tom-gawra|@list.ru

\author{
Кладиев Сергей Николаевич', \\ kladiev@tpu.ru \\ Глазырин Александр Савельевич', \\ asglazyrin@tpu.ru
}

\author{
Боловин Евгений Владимирович', \\ bolovinev@mail.ru
}

\author{
Полищук Владимир Иосифович ${ }^{2}$ \\ polischuk_vi@mail.ru \\ 1 Национальный исследовательский Томский политехнический университет, \\ Россия, 635050, г. Томск, пр. Ленина, 30. \\ ${ }^{2}$ Алтайский государственный технический университет им. И.И. Ползунова, \\ Россия, 656038, г. Барнаул, пр. Ленина, 46.
}

\begin{abstract}
Актуальность исследования обусловлена тем, что вибрационные электромагнитные активаторы являются эффективными устройствами для перемешивания суспензий, эмульсий, приготовления буровых растворов, разжижения высоковязких нефтепродуктов. Якорь специальной конструкции представляет собой гидравлический вентиль. При вибрации якоря на частотах, близких к резонансной частоте, в обрабатываемой жидкой среде создаются глубоко затопленные струи, которые при относительно невысоком энергопотреблении вибрационного электромагнитного активатора обеспечивают высокую эффективность перемешивания жидкой среды и снижение вязкости нефтепродуктов на продолжительном интервале времени. Резонансная частота механической системы зависит от жесткости пружины, массы якоря-активатора, присоединенной массы колеблющейся с якорем жидкой среды и от коэффициента вязкого трения, определяющего отведение энергии из колеблющейся механической системы. При изменении реологических свойств обрабатываемой жидкой среды изменяются как параметры механической колебательной системы, так и вид амплитудно-частотных характеристик вибрационного электромагнитного активатора. Способ организации мониторинга изменения реологических свойств обрабатываемой вибрационным электромагнитным активатором жидкой среды на основе прямых измерений, например с помощью вискозиметров, пригоден только для лабораторных условий и не годится для промышленного внедрения. По мнению авторов, более перспективным является подход, основанный на решении обратной математической задачи, когда, анализируя вид амплитудно-частотных характеристик вибрационного электромагнитного активатора, в частности граничные околорезонансные частоты, можно получить достоверные оценки параметров механической колебательной системы вибрационного электромагнитного активатора. Эти оценки удобно применять для организации как косвенного мониторинга изменения реологических свойств обрабатываемой жидкости в процессе работы вибрационного электромагнитного активатора, так и для усовершенствования структуры системы автоматического управления вибрационным электромагнитным активатором.
\end{abstract}

Цель исследования заключается в разработке метода идентификации параметров механической системы вибрационного электромагнитного активатора на основе анализа граничных околорезонансных частот и определении границ применимости метода в сильно нагруженных колебательных механических системах.

Методы: обыкновенные дифференциальные уравнения, преобразование Лапласа, передаточные функции, амплитудно-частотные характеристики, алгебраические уравнения.

Результаты. Получены аналитические выражения, связывающие граничные околорезонансные частоты с параметрами механической колебательной системы, на основе которых составляются системы алгебраических уравнений. Показаны границы применимости метода в сильно нагруженных колебательных механических системах.

\section{Ключевые слова:}

Вибрационный электромагнитный активатор, механическая система, параметры, идентификация, резонанс, амплитудно-частотная характеристика, граничные околорезонансные частоты.

\section{Введение}

Электромагнитные двигатели с возвратно-поступательным движением [1-3] находят применение в технологиях, связанных с сейсморазведкой [4-9], приготовлением буровых растворов [10, 11], подготовкой высоковязких нефтепродуктов к транспортировке [12-17]. Работающие в околоре- зонансном режиме вибрационные электромагнитные активаторы в качестве разжижителей высоковязких нефтепродуктов имеют ряд преимуществ [18] в массогабаритных показателях и энергопотреблении по сравнению с винтовыми устройствами размыва донных отложений, что позволяет более удобно встраивать вибрационные электромаг- 
нитные активаторы (ВЭМА) в технологический процесс [19, 20]. Для эффективного управления ВЭМА требуется автоматическая подстройка $[21,22]$ на резонансную частоту и предельный безударный режим работы [23], что можно обеспечить на основе непрерывного косвенного мониторинга изменения реологических свойств обрабатываемой жидкости, которые непосредственно связаны с параметрами механической колебательной системы.

Предложенный способ идентификации параметров механической системы вибровозбудителя, работающего в околорезонансном диапазоне частот, позволяет определить диссипативные параметры колебательной системы путем анализа вида амплитудно-частотных характеристик. Полученные оценки позволяют косвенно оценить вязкость обрабатываемой жидкости.

В представленной работе проведено аналитическое исследование частотных характеристик ВЭМА в рабочем диапазоне частот, при условии, что удары якоря-активатора о стенки корпуса отсутствуют, т. е. наблюдается режим безударной работы. Для настройки на резонансную частоту могут использоваться датчики ускорения, скорости или положения, поэтому было бы логично проанализировать частотные характеристики механического канала ВЭМА для ускорения, скорости и отклонения якоря от положения равновесия (виброперемещения). Исследовалась механическая система ВЭМА с «глухим якорем», результаты же в основном применимы для электромеханического преобразователя с виброструйным якорем-активатором. Показано влияние непостоянства присоединённой колеблющейся массы и ослабления пружины на частотные характеристики.

\section{Математическая модель для описания взаимосвязи между параметрами механического колебательного контура и граничными околорезонанстными частотами}

Нижеприведенные выкладки по описанию математической модели, позволяющей находить взаимосвязи между параметрами механического колебательного контура ВЭМА и граничными околорезонанстными частотами, составлялись при условии ее линеаризации и стационарности параметров в течении всего промежутка времени измерения и построения амплитудно-частотных характеристик (АЧХ). Вопрос о погрешности линеаризации математической модели механической колебательной системы ВЭМА рассмотрен в [19], где было отмечено, что на основании сопоставления полученных на имитационной модели и экспериментальных АЧХ ВЭМА погрешность настройки на резонансную частоту с использованием такой линеаризованной модели не превышает $6 \%$, что является допустимым. Для заявленных целей, а именно для мониторинга изменения реологических свойств обрабатываемой жидкой среды и самонастройки автоматической системы управления ВЭМА, такой подход является оправданным.
В статье все физические величины приведены в единицах СИ по умолчанию, кроме оговоренных особо.

Запишем уравнение равновесия для механического контура ВЭМА:

$$
F_{\text {эм }}(t)=m_{\Sigma} \frac{d^{2}}{d t^{2}} x(t)+R_{\text {мех }} \frac{d}{d t} x(t)+x(t) k_{\text {пр }},
$$

где $F_{\text {эм }}(t)$ - сила, стягивающая магнитный зазор, является вынуждающей; $x(t)$ - отклонение активатора от положения равновесия; $R_{\text {мех }}=100 \mathrm{\kappa г} / \mathrm{c}-$ коэффициент вязкого трения; $k_{\text {пр }}=1,85 \cdot 10^{5} \mathrm{H} / \mathrm{м}-$ жёсткость пружины; $m_{\Sigma}=1,157$ кг - суммарная колеблющаяся масса $m_{\Sigma}=m_{\text {a }}+m_{\text {присед}}$, состоит из $m_{\mathrm{a}}=0,49$ кг - массы якоря-активатора и $m_{\text {присоед }}$ присоединённой колеблющейся массы жидкости.

Согласно принятому допущению о линейности и стационарности математической модели механического контура ВЭМА(1), будем считать, что суммарная колеблющаяся масса, жёсткость пружины и коэффициент вязкого трения не зависят от $x(t)$ и его производных $\frac{d x(t)}{d t}$ и $\frac{d^{2} x(t)}{d t^{2}}$, а также от времени, следовательно, правомерно применять к уравнению (1) любые временные и частотные преобразования.

Применив преобразование Лапласа, получим:

$$
\begin{gathered}
F_{\text {эм }}(p)=m_{\Sigma} x(p) p^{2}+R_{\text {мех }} x(p) p+k_{\text {пр }} x(p) ; \\
F_{\text {эм }}(p)=x(p)\left(m_{\Sigma} p^{2}+R_{\text {мех }} p+k_{\text {пр }}\right) ; \\
F_{\text {эм }}(p)=m_{\Sigma} x(p)\left(p^{2}+\frac{R_{\text {мех }}}{m_{\Sigma}} p+\frac{k_{\text {пр }}}{m_{\Sigma}}\right),
\end{gathered}
$$

где $p$ - оператор Лапласа.

Для адекватной оценки вибрационных воздействий на систему измеряются параметры вибрации (форма сигнала, спектр). Оценка обычно производится на соответствующих частотах: 10-100 Гц по величине размаха виброперемещений, 100-1000 Гц по величине виброскорости, свыше 1000 Гц по значению виброускорения [24, 25]. Как правило, в качестве датчиков используются акселерометры, сигнал от которых посредством процедуры интегрирования превращается в необходимые физические величины виброскорости или виброперемещения. Поэтому в данной статье будут рассмотрены передаточные функции и АЧХ для каждого из указанных видов сигналов.

В случае если для измерения параметров вибрации используется сигнал от датчика виброперемещения, передаточная функция механического контура ВЭМА - отношение изображений по Лапласу величины отклонения активатора от положения равновесия к вызвавшей это отклонение электромагнитной силе

$$
W_{\text {мех }}(p)=\frac{x(p)}{F_{\text {эм }}(p)}=\frac{1}{\left(m_{\Sigma} p^{2}+R_{\text {мех }} p+k_{\text {пр }}\right)} .
$$

Амплитуда $x(p)$ пропорциональна произведению $F_{\text {эм }}(p)$ на $W_{\text {мех }}(p)$ 


$$
x(p)=\frac{F_{\text {эм }}(p)}{\left(m_{\Sigma} p^{2}+R_{\text {мех }} p+k_{\text {пр }}\right)}=F_{\text {эм }}(p) W_{\text {мех }}(p) .
$$

Сделаем подстановку $p=j \omega$, где $j=\sqrt{-1}-$ мнимая единица; $\omega=2 \pi f$ - круговая частота; $f$ - циклическая частота, получим $W_{\text {мех }}(j \omega)$ и представим её вещественной $P(\omega)$ и мнимой $Q(\omega)$ составляющими

$$
\begin{array}{r}
W_{\text {mex }}(j \omega)=\frac{1}{\left(m_{\Sigma} j^{2} \omega^{2}+R_{\text {mex }} j \omega+k_{\text {mp }}\right)} ; \\
W_{\text {mex }}(j \omega)=\frac{1}{\left(-m_{\Sigma} \omega^{2}+R_{\text {mex }} j \omega+k_{\text {mp }}\right)}= \\
=\frac{1}{\left(k_{\text {mp }}-m_{\Sigma} \omega^{2}\right)+R_{\text {mex }} j \omega} ; \\
W_{\text {mex }}(j \omega)=\frac{\left(k_{\text {пр }}-m_{\Sigma} \omega^{2}\right)-R_{\text {мex }} j \omega}{\left[\left(k_{\text {пр }}-m_{\Sigma} \omega^{2}\right)+R_{\text {мех }} j \omega\right]} \times \\
\times\left[\left(k_{\text {пр }}-m_{\Sigma} \omega^{2}\right)-R_{\text {mex }} j \omega\right]^{-1} .
\end{array}
$$

В итоге после выполнения стандартной операции домножения на комплексно-сопряженную компоненту получим механическую комплексную частотную характеристику:

$$
W_{\text {мех }}(j \omega)=\frac{\left(k_{\text {пр }}-m_{\Sigma} \omega^{2}\right)-R_{\text {мех }} j \omega}{\left(k_{\text {пр }}-m_{\Sigma} \omega^{2}\right)^{2}+R_{\text {мex }}^{2} \omega^{2}} ;
$$

Механическая вещественная частотная характеристика

$$
P(\omega)=\frac{k_{\text {пр }}-m_{\Sigma} \omega^{2}}{\left(k_{\text {пр }}-m_{\Sigma} \omega^{2}\right)^{2}+R_{\text {мex }}^{2} \omega^{2}} .
$$

Механическая мнимая частотная характеристика

$$
Q(\omega)=\frac{-R_{\text {мex }} \omega}{\left(k_{\text {пр }}-m_{\Sigma} \omega^{2}\right)^{2}+R_{\text {мex }}^{2} \omega^{2}} .
$$

Механическая амплитудно-частотная характеристика

$$
\begin{aligned}
A(\omega) & =\left|W_{\text {мех }}(j \omega)\right|=\sqrt{P(\omega)^{2}+Q(\omega)^{2}}= \\
& =\frac{\sqrt{\left(k_{\text {mp }}-m_{\Sigma} \omega^{2}\right)^{2}+\left(-R_{\text {mex }} \omega\right)^{2}}}{\left(k_{\text {mp }}-m_{\Sigma} \omega^{2}\right)^{2}+R_{\text {мex }}^{2} \omega^{2}} ; \\
A(\omega) & =\frac{1}{\sqrt{k_{\text {mp }}^{2}-2 k_{\text {пр }} m_{\Sigma} \omega^{2}+m_{\Sigma}^{2} \omega^{4}+R_{\text {mex }}^{2} \omega^{2}}} .
\end{aligned}
$$

При $\omega \rightarrow 0$ амплитуда виброперемещения стремится к пределу

$$
\lim \underset{\omega \rightarrow 0}{A(\omega)}=\frac{1}{k_{\text {пр }}} .
$$

Приравняв числитель производной механической амплитудно-частотной характеристики по частоте

$$
\frac{d A(\omega)}{d \omega}=\frac{-1\left(-4 k_{\text {пр }} m_{\Sigma} \omega+4 m_{\Sigma}^{2} \omega^{3}+2 R_{\text {мех }}^{2} \omega\right)}{2\left(k_{\text {mp }}^{2}-2 k_{\text {mp }} m_{\Sigma} \omega^{2}+m_{\Sigma}^{2} \omega^{4}+R_{\text {мex }}^{2} \omega^{2}\right)^{\frac{3}{2}}}
$$

к нулю

$$
-4 k_{\text {пр }} m_{\Sigma} \omega+4 m_{\Sigma}^{2} \omega^{3}+2 R_{\text {мех }}^{2} \omega=0,
$$

получим уравнение, решив которое, найдём три корня - два побочных

$$
\omega_{01}=0 \text { и } \omega_{02}=-\sqrt{\frac{k_{\text {mp }}}{m_{\Sigma}}-\left(\frac{R_{\text {мex }}}{2 m_{\Sigma}}\right)^{2}},
$$

и один основной корень, который является частотой свободных колебаний или резонансной частотой механического контура ВЭМА

$$
\omega_{0}=\sqrt{\frac{k_{\text {пр }}}{m_{\Sigma}}-\left(\frac{R_{\text {мex }}}{2 m_{\Sigma}}\right)^{2}} .
$$

Граничная величина коэффициента вязкого трения $R_{\text {мех_гр }}=2 \sqrt{k_{\text {пp }} m_{\Sigma}}$.

Вид семейств АЧХ механического контура ВЭМА при варьировании параметров представлен на рис. 1.

При стремлении вынуждающей частоты к частоте свободных колебаний механического контура амплитуда виброперемещения стремится к максимуму $A_{\max }$, величина которого зависит от параметров механического контура

$$
\begin{aligned}
& A_{\text {max }}=\lim A(\omega)= \\
& =\left[\begin{array}{l}
\left(k_{\text {пр }}-m_{\Sigma}\left(\frac{k_{\text {пр }}}{m_{\Sigma}}-\left(\frac{R_{\text {мех }}}{2 m_{\Sigma}}\right)^{2}\right)\right)^{2}+ \\
+R_{\text {мех }}^{2}\left(\frac{k_{\text {пр }}}{m_{\Sigma}}-\left(\frac{R_{\text {мех }}}{2 m_{\Sigma}}\right)^{2}\right)
\end{array}\right]^{\frac{-1}{2}}
\end{aligned}
$$

В работе [26] граничные околорезонансные частоты механического контура ВЭМА было предложено описывать стандартным способом, принятым в теории фильтров [27]. Этот подход заключался в том, что граничные околорезонасные частоты находились из решения нелинейного алгебраического уравнения [28-30], которое составлялось с учетом того, что амплитуда виброперемещения $A(\omega)$ на граничных околорезонансных частотах уменьшается в $\sqrt{2}$ раз $A_{\max }=\underset{\omega \rightarrow \omega_{\mathrm{m} \_} 1}{A}=\underset{\omega \rightarrow \omega_{\mathrm{m} L 2}}{A}$, где $\omega_{\mathrm{mI} \_1}-$ нижняя граничная частота полосы пропускания механической амплитудно-частотной характеристики; $\omega_{\text {пा_2 }}-$ верхняя граничная частота полосы пропускания механической амплитудно-частотной характеристики.

Такой подход хорошо себя зарекомендовал и апробирован на основе экспериментальных данных в слабодемфированных колебательных механических системах $[19,20]$, для которых справедливо неравенство $\frac{A\left(\omega_{0}\right)}{\sqrt{2}}>A(0)$. Но идею о возможности нахождения параметров механической колебательной системе по граничным околорезонансным частотам $\omega_{\text {II__1 }}$ и $\omega_{\text {m__2 }}$ можно распространить 

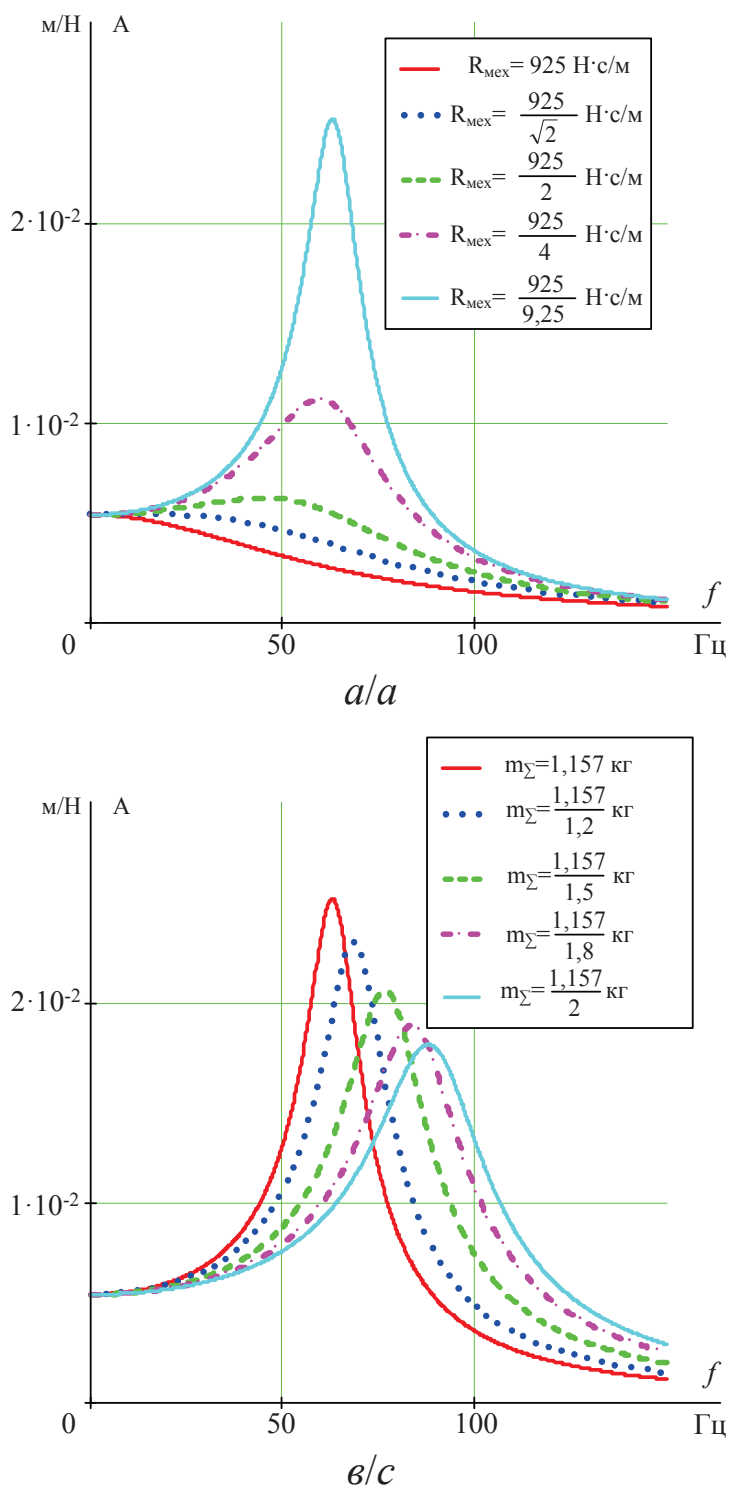

и на механические системы с большей степенью демпфирования. Для этого амплитуда виброперемещения $A(\omega)$ на граничных околорезонансных частотах будет определяться на основе следующего нелинейного алгебраического уравнения

$$
\frac{A\left(\omega_{0}\right)-A(0)}{2}=\underset{\omega \rightarrow \omega_{\mathrm{mL} \_1}}{A}(\omega) \underset{\omega \rightarrow \omega_{\mathrm{m} \_\_}}{A}(\omega) .
$$

Для нахождения этих частот с учётом (2) составим равенство

$$
\begin{aligned}
& \frac{1}{\sqrt{\left(k_{\text {пр }}-m_{\Sigma} \omega^{2}\right)^{2}+R_{\text {Mex }}^{2} \omega^{2}}}= \\
& =\frac{1}{2}\left\{\frac{1}{k_{\text {пр }}}+\left[\begin{array}{l}
\left(k_{\text {пр }}-m_{\Sigma}\left(\frac{k_{\text {пр }}}{m_{\Sigma}}-\left(\frac{R_{\text {мех }}}{2 m_{\Sigma}}\right)^{2}\right)\right)^{2}+ \\
+R_{\text {мех }}^{2}\left(\frac{k_{\text {пр }}}{m_{\Sigma}}-\left(\frac{R_{\text {мех }}}{2 m_{\Sigma}}\right)^{2}\right)
\end{array}\right]^{\frac{-1}{2}}\right\} .
\end{aligned}
$$

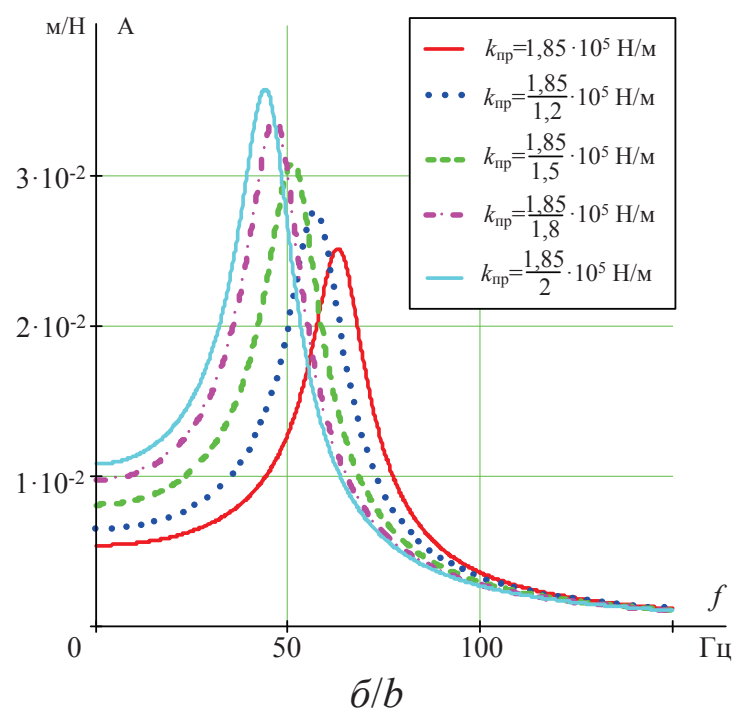

Pис.1. Алплитудно-частотные характеристики ВЭМА по виброперемещения при варьировании параметров: а) коэффициента вязкого трения; б) жёсткости пружины; в) суммарной колеблющейся массы

Fig. 1. Frequency response of vibration electromagnetic activator (VEMA) by vibration displacement with varying parameters: a) viscous friction coefficient; $b$ ) spring stiffness c) total oscillating mass

Обозначив правую часть вышеприведенного равенства как

$$
a a=\frac{1}{2}\left\{\frac{1}{k_{\text {пр }}}+\left[\begin{array}{l}
\left.\left(k_{\text {пр }}-m_{\Sigma}\left(\frac{k_{\text {пр }}}{m_{\Sigma}}-\left(\frac{R_{\text {мех }}}{2 m_{\Sigma}}\right)^{2}\right)\right)^{2}+\right]^{\frac{-1}{2}} \\
+R_{\text {мех }}^{2}\left(\frac{k_{\text {пр }}}{m_{\Sigma}}-\left(\frac{R_{\text {мех }}}{2 m_{\Sigma}}\right)^{2}\right)
\end{array}\right\},\right.
$$

получим уравнение четвёртого порядка

$$
\frac{1}{\sqrt{\left(k_{\text {пр }}-m_{\Sigma} \omega^{2}\right)^{2}+R_{\text {мех }}^{2} \omega^{2}}}-a a=0,
$$

решив которое, найдём четыре корня.

Уравнение (3) имеет два корня: положительный и отрицательный. Отрицательный корень отбрасываем. В итоге, решив уравнение (3), получим четыре корня - два положительных и два отрицательных. Меньший из положительных корней ура- 
внения (3) является нижней граничной частотой полосы пропускания механической амплитудночастотной характеристики (рис. 2,3$)$

$$
\omega_{\text {пा_ } \_1}=
$$

$$
=\left[\begin{array}{l}
-\frac{\left(a a^{2} R_{\mathrm{mex}}^{2}-4 k_{\mathrm{mp}} a a^{2} m_{\Sigma} R_{\mathrm{mex}}+4 m_{\Sigma}{ }^{2}\right)^{\frac{1}{2}}}{2 a a m_{\Sigma}{ }^{2}}+ \\
+\frac{a a R_{\mathrm{mex}}-2 a a m_{\Sigma} k_{\mathrm{mp}}}{2 a a m_{\Sigma}^{2}}
\end{array}\right]^{\frac{1}{2}},
$$

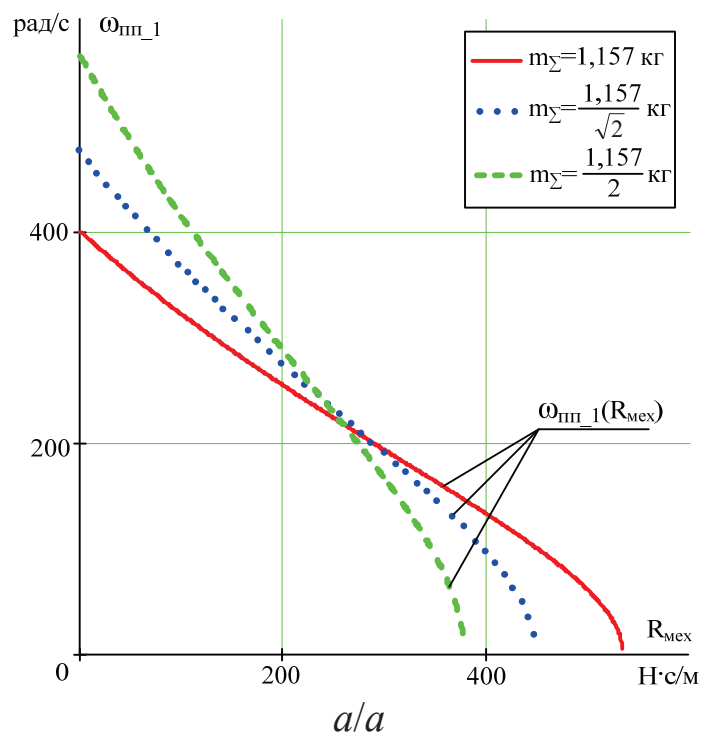

а больший из положительных корней уравнения (3) является верхней граничной частотой полосы пропускания механической амплитудно-частотной характеристики (рис. 4,5$)$

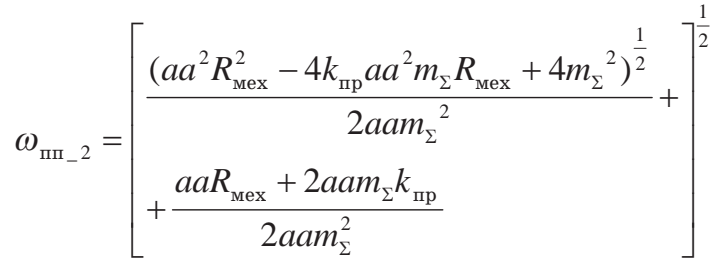

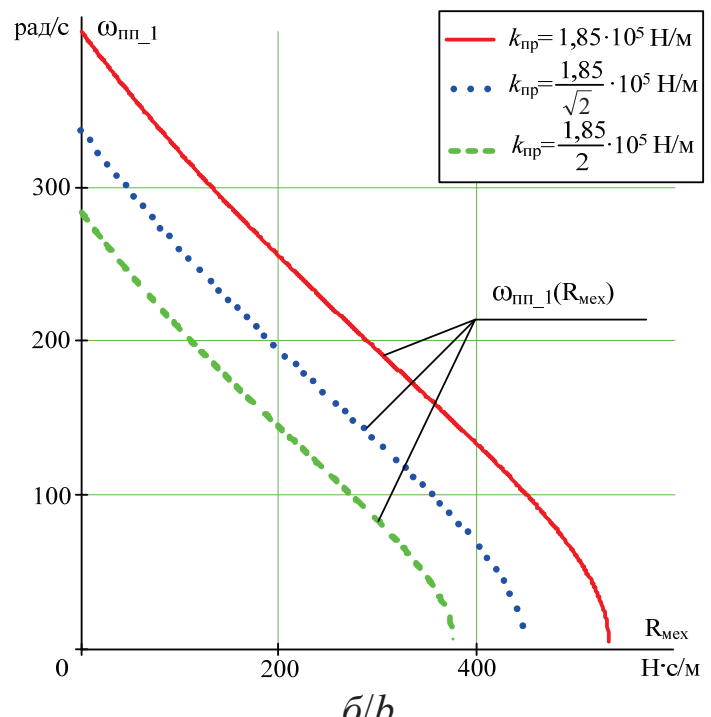

Pис. 2. Зависимость нижней граничной частоты полосы пропускания АЧХ ВЭМА по виброперелещению от коэффициента вязкого трения при варьировании параметров: а) сумларной колеблющейся массы; б) жёсткости пружины

Fig. 2. Dependence of the lower limiting frequency of the VEMA frequency response by vibration displacement on the viscous friction coefficient with varying parameters: a) total oscillating mass; b) spring stiffness
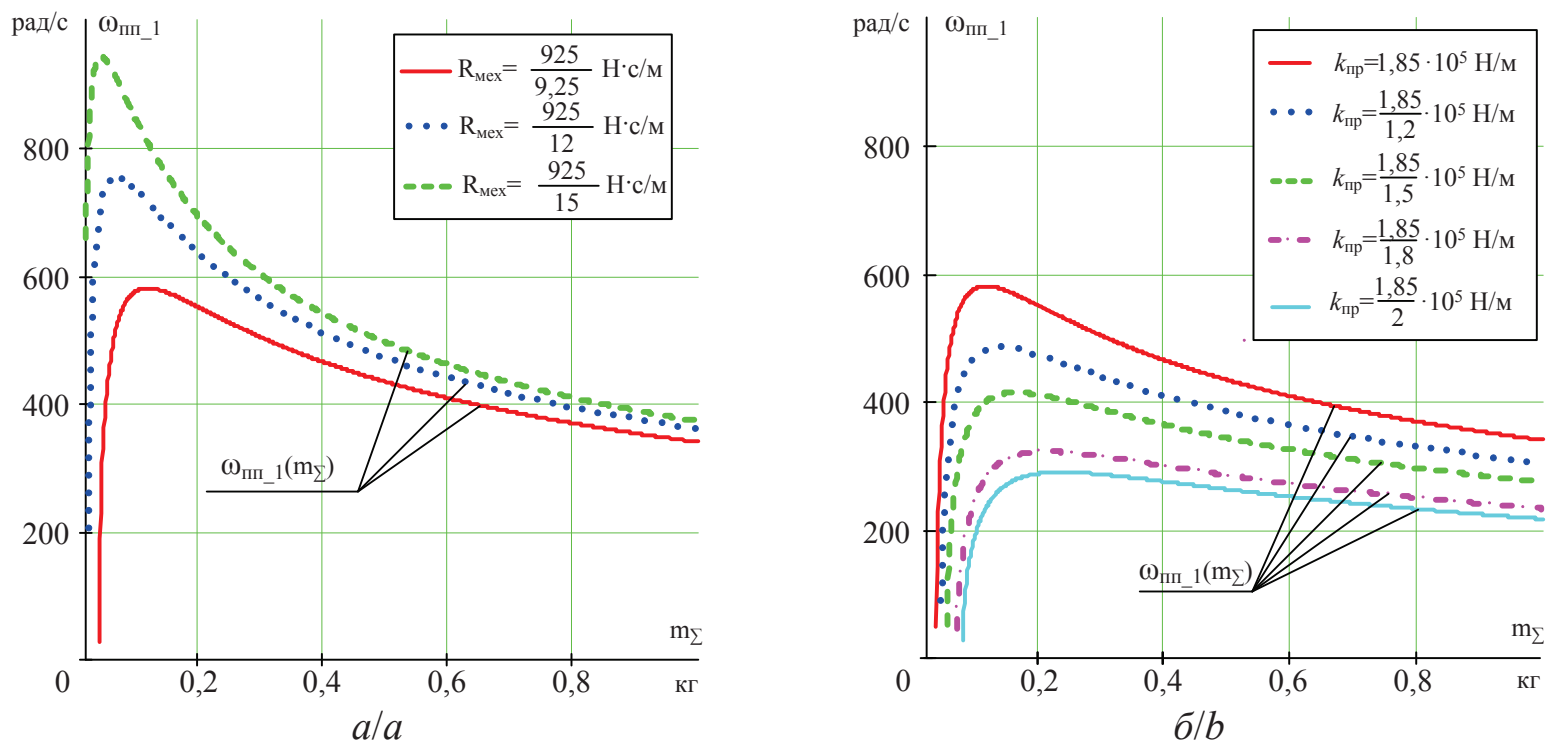

Pис. 3. Зависимость нижней граничной частоты полосы пропускания АЧХ ВЭМА по виброперемещению от сумларной колеблющейся массы при варьировании параметров: а) коэффициента вязкого трения; б) жёсткости пружины

Fig. 3. Dependence of the lower limiting frequency of the VEMA frequency response by vibration displacement on the total oscillating mass with varying parameters: a) total viscous friction coefficient; $b$ ) spring stiffness 

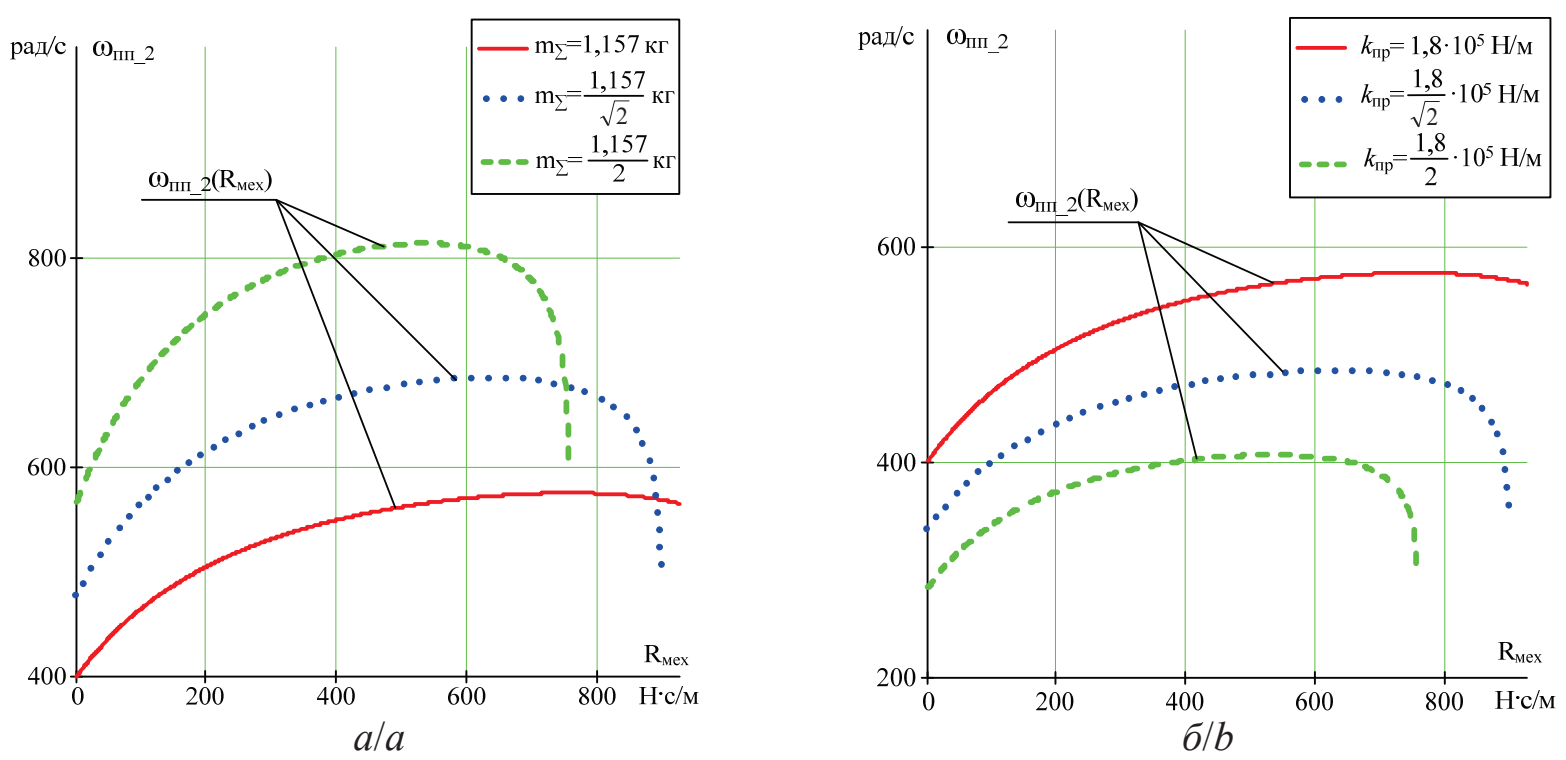

Pис.4. Зависилость верхней граничной частоты полосы пропускания АЧХ ВЭМА по виброперемещению от коэффициента вязкого трения при варьировании параметров: а) сумларной колеблющейся массы; б) жёсткости пружины

Fig. 4. Dependence of the higher limiting frequency of the VEMA frequency response by vibration displacement on the viscous friction coefficient with varying parameters: a) total oscillating mass; $b$ ) spring stiffness
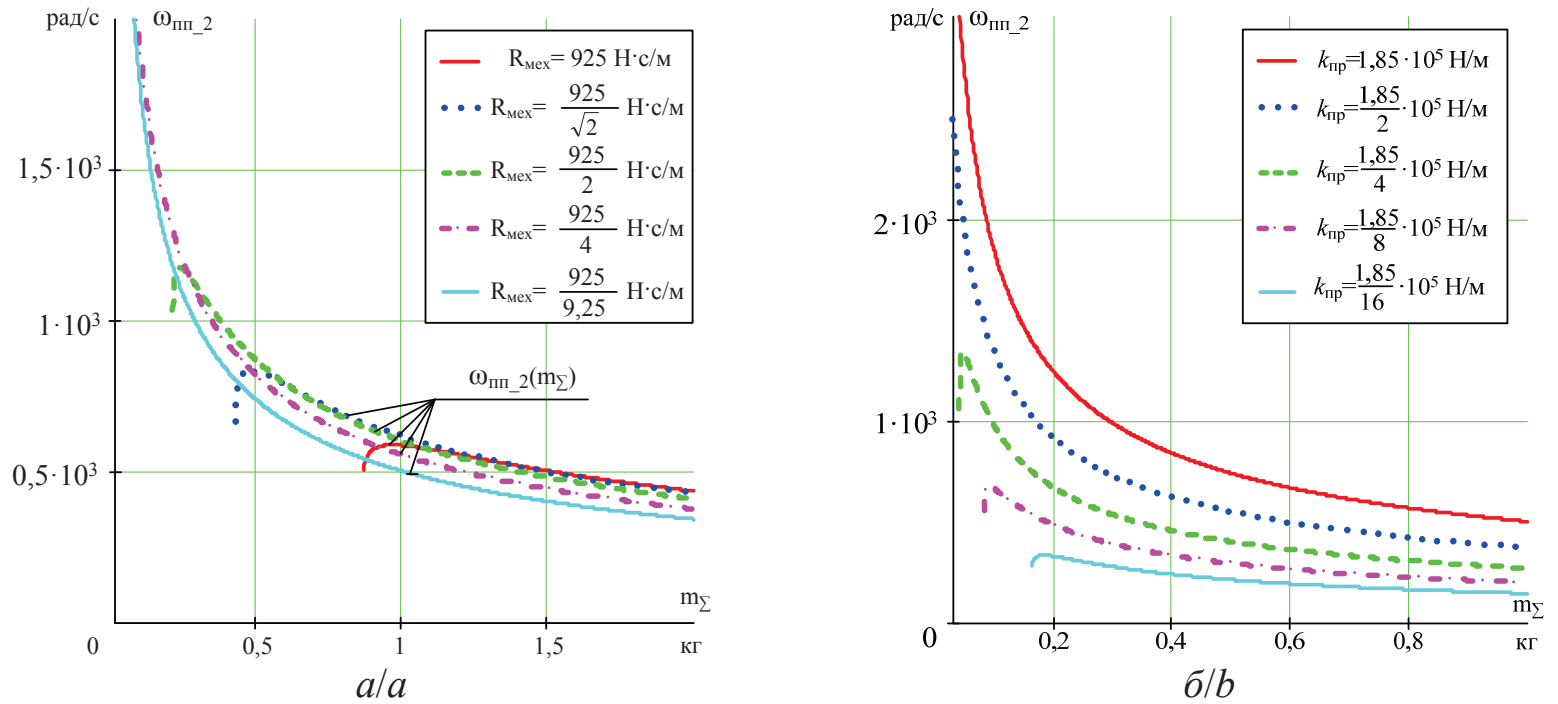

Pис. 5. Зависимость верхней граничной частоты полосы пропускания АЧХ ВЭМА по виброперемещению от суммарной колеблющейся массы при варьировании параметров: а) коэффициента вязкого трения; б) жёсткости пружины.

Fig. 5. Dependence of the higher limiting frequency of the VEMA frequency response by vibration displacement on the total oscillating mass with varying parameters: a) total viscous friction coefficient; $b$ ) spring stiffness

Получены зависимости граничных частот полосы пропускания механической амплитудно-частотной характеристики от суммарной колеблющейся массы и коэффициента вязкого трения. Зная эти граничные частоты, можно решить систему нелинейных уравнений (4) и (5) и определить действующие значения присоединённой колеблющейся массы и коэффициент вязкого трения.

Имея частоты, ограничивающие полосу пропускания, определяемые по (4) и (5), можно вывести выражение для ширины полосы пропускания, которая, в свою очередь, тоже зависит от суммарной колеблющейся массы и коэффициента вязкого тре- ния $\Delta \omega_{\text {mII }}=\omega_{\text {프.2 }}-\omega_{\text {mII_1. }}$. Соответствующие аналитические выражения из-за ограниченности объема статьи здесь не приводятся, но их можно вывести с использованием выражений (4) и (5).

Ширина полосы пропускания прямо пропорциональна коэффициенту вязкого трения, в то же время обратно пропорциональна суммарной колеблющейся массе при больших массах и прямо пропорциональна при малых массах (рис. 6,7 ).

В случае если для измерения параметров вибрации используется сигнал от датчика виброскорости, передаточная функция механического контура по скорости - отношение величины виброскоро- 

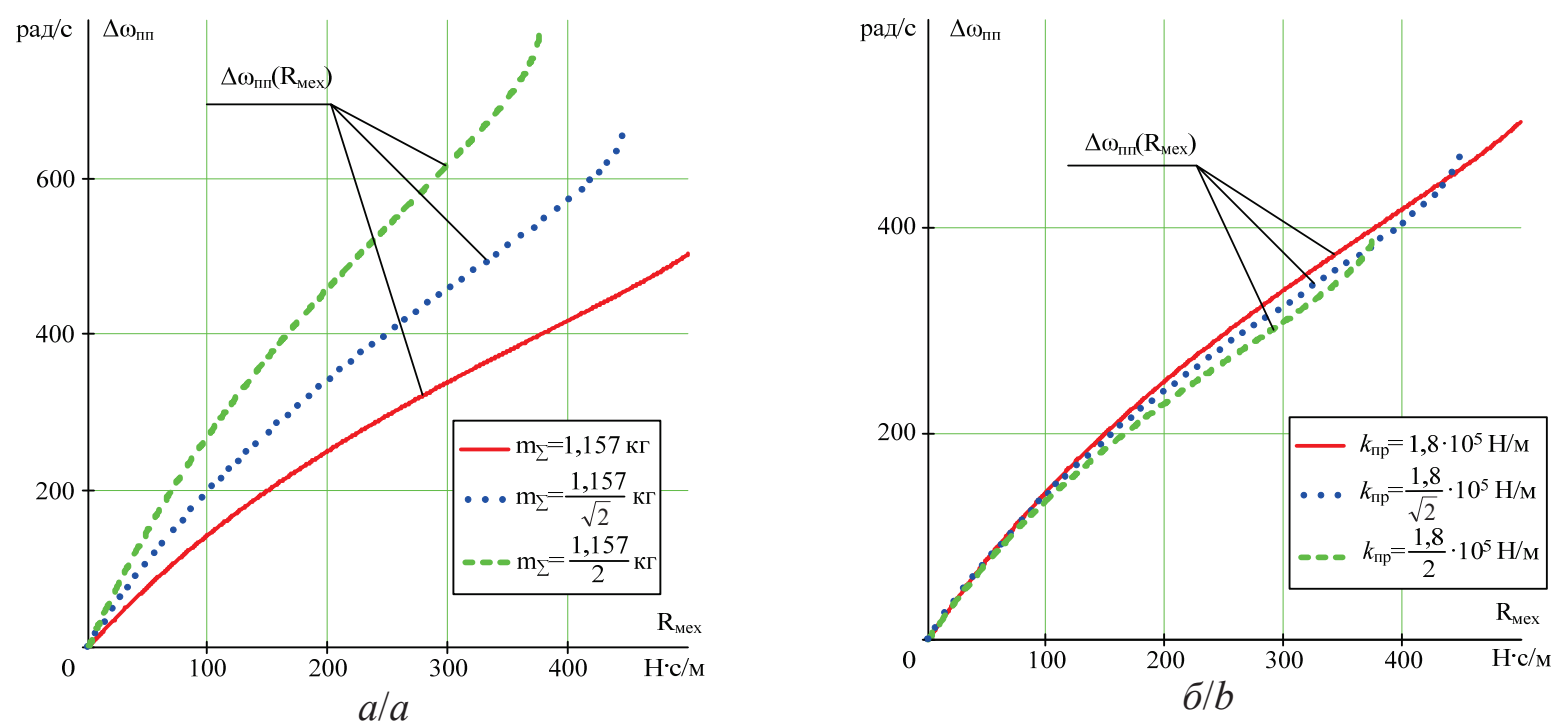

Рис. 6. Зависимость ширины полосы пропускания АЧХ ВЭМА по виброперемещению от коэффициента вязкого трения при варьировании параметров: а) сумларной колеблющейся массы; б) жёсткости пружины

Fig. 6. Dependence of the bandwidth of the VEMA frequency response by vibration displacement on the viscous friction coefficient with varying parameters: a) total oscillating mass; b) spring stiffness
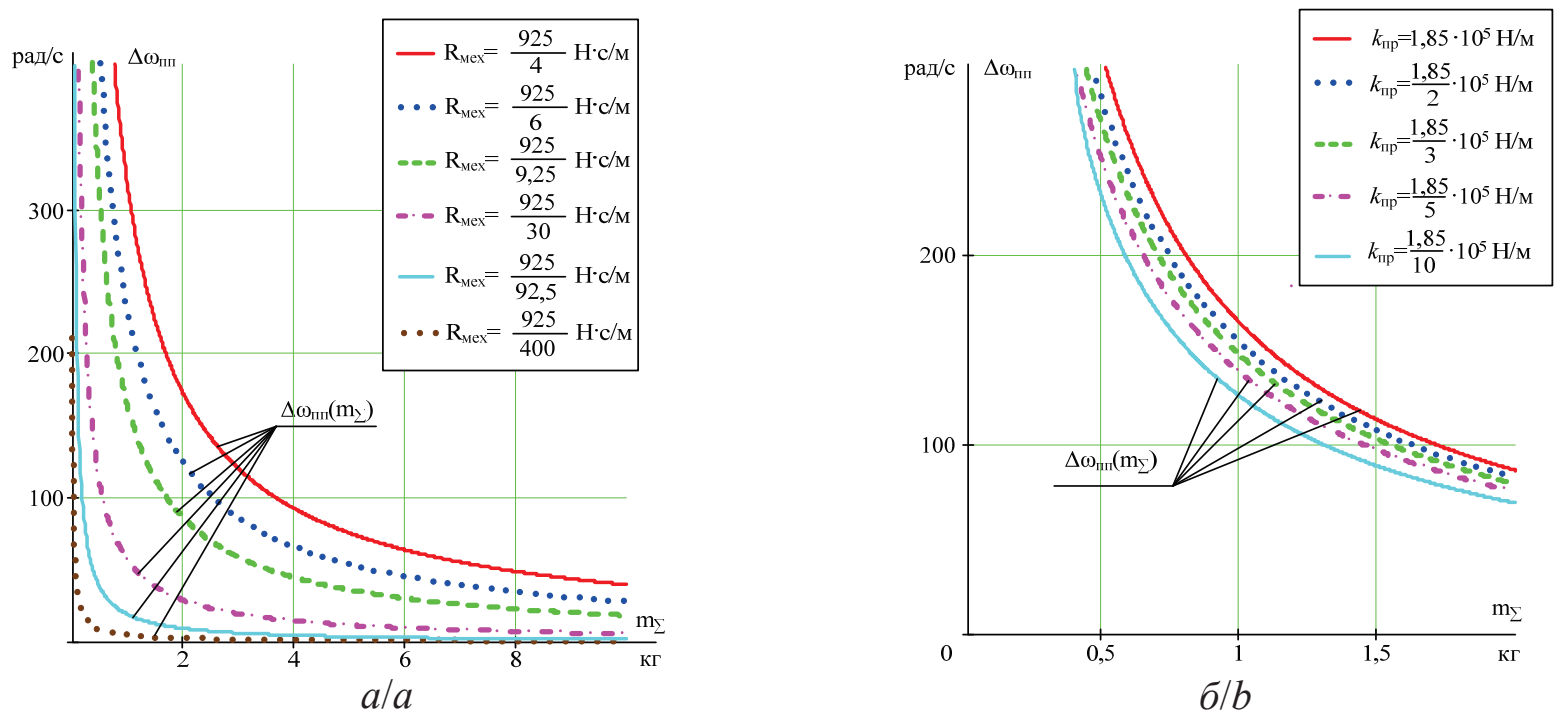

Рис. 7. Зависилость ширины полосы пропускания АЧХ ВЭМА по виброперемещению от сулмарной колеблющейся массы при варьировании параметров: а) коэффициента вязкого трения; б) жёсткости пружины.

Fig. 7. Dependence of the bandwidth of the VEMA frequency response by vibration displacement on the total oscillating mass with varying pa rameters: a) total viscous friction coefficient; b) spring stiffness

сти к приложенной к механическому контуру электромагнитной силе

$$
W_{V}(p)=\frac{V(p)}{F_{\text {эм }}(p)}=\frac{p}{m_{\Sigma} p^{2}+R_{\text {мех }} p+k_{\text {пр }}} .
$$

Амплитуда виброскорости $V(p)$ пропорциональна произведению $F_{\text {эм }}(p)$ на $W_{V}(p)$

$$
V(p)=\frac{F_{\text {эм }}(p) p}{m_{\Sigma} p^{2}+R_{\text {мех }} p+k_{\text {пр }}}=F_{\text {эм }}(p) W_{V}(p) .
$$

После подстановки $p=j \omega$ получим $W_{V}(j \omega)$ и представим её составляющими $P_{V}(\omega)$ и $Q_{V}(\omega)$

$$
\begin{gathered}
W_{V}(j \omega)=\frac{j \omega}{m_{\Sigma}(j \omega)^{2}+R_{\text {мех }} j \omega+k_{\text {пр }}}= \\
=\frac{j \omega}{\left(k_{\text {пр }}-m_{\Sigma} \omega^{2}\right)+R_{\text {мех }} j \omega} ; \\
W_{V}(j \omega)=\frac{j \omega\left[\left(k_{\text {пр }}-m_{\Sigma} \omega^{2}\right)-R_{\text {мех }} j \omega\right]}{\left(k_{\text {пр }}-m_{\Sigma} \omega^{2}\right)+R_{\text {мех }} j \omega} \times \\
\times\left[\left(k_{\text {пр }}-m_{\Sigma} \omega^{2}\right)-R_{\text {мех }} j \omega\right]^{-1} ; \\
W_{V}(j \omega)=\frac{j \omega\left[\left(k_{\text {пр }}-m_{\Sigma} \omega^{2}\right)-R_{\text {мех }} j \omega\right]}{\left(k_{\text {пр }}-m_{\Sigma} \omega^{2}\right)^{2}+R_{\text {мех }}^{2} \omega^{2}} .
\end{gathered}
$$



стика

Скоростная комплексно-частотная характери-

$$
\begin{gathered}
W_{V}(j \omega)=\frac{R_{\text {мех }} \omega^{2}+j \omega\left(k_{\text {пр }}-m_{\Sigma} \omega^{2}\right)}{\left(k_{\text {пр }}-m_{\Sigma} \omega^{2}\right)^{2}+R_{\text {мех }}^{2} \omega^{2}} ; \\
W_{V}(j \omega)=\frac{R_{\text {мех }} \omega^{2}}{\left(k_{\text {пр }}-m_{\Sigma} \omega^{2}\right)^{2}+R R_{\text {мех }}^{2} \omega^{2}}+ \\
+\frac{j \omega\left(k_{\text {пр }}-m_{\Sigma} \omega^{2}\right)}{\left(k_{\text {пр }}-m_{\Sigma} \omega^{2}\right)^{2}+R_{\text {мех }}^{2} \omega^{2}} .
\end{gathered}
$$

Скоростная вещественная частотная характеристика

$$
P_{V}(\omega)=\frac{R_{\text {мех }} \omega^{2}}{\left(k_{\text {пр }}-m_{\Sigma} \omega^{2}\right)^{2}+R_{\text {мех }}^{2} \omega^{2}} .
$$

Скоростная мнимая частотная характеристика

$$
Q_{V}(\omega)=\frac{\omega\left(k_{\text {пр }}-m_{\Sigma} \omega^{2}\right)}{\left(k_{\text {пр }}-m_{\Sigma} \omega^{2}\right)^{2}+R_{\text {мех }}^{2} \omega^{2}} .
$$
стика

Скоростная амплитудно-частотная характери-

$$
\begin{gathered}
A_{V}(\omega)=\left|W_{V}(j \omega)\right|=\sqrt{P_{V}(\omega)^{2}+Q_{V}(\omega)^{2}}= \\
=\frac{\sqrt{\left(R_{\text {мех }} \omega^{2}\right)^{2}+\left[\omega\left(k_{\text {пр }}-m_{\Sigma} \omega^{2}\right)\right]^{2}}}{\left(k_{\text {пр }}-m_{\Sigma} \omega^{2}\right)^{2}+R_{\text {мех }}^{2} \omega^{2}} ; \\
A_{V}(\omega)=\frac{\left(R_{\text {мех }}^{2} \omega^{4}+\omega^{2} k_{\text {пр }}^{2}-2 \omega^{4} k_{\text {пр }} m_{\Sigma}+\omega^{6} m_{\Sigma}{ }^{2}\right)^{\frac{1}{2}}}{\left(k_{\text {пр }}-m_{\Sigma} \omega^{2}\right)^{2}+R_{\text {мех }}^{2} \omega^{2}} .
\end{gathered}
$$

При стремлении вынуждающей частоты к частоте свободных колебаний механического контура амплитуда виброскорости аналогично стремится к максимуму, величина которого зависит от параметров механического контура (рис. 8)

$$
\begin{aligned}
& A_{V}\left(\omega_{0}\right)=\underset{\omega=\omega_{0}}{A}(\omega)=\frac{A_{V}(\omega) ;}{\omega=\sqrt{\frac{k_{\text {mp }}}{m_{\Sigma}}-\left(\frac{R_{\text {sex }}}{2 m_{\Sigma}}\right)^{2}}} ; \\
& A_{v}\left(\omega_{0}\right)=\left\{\left[k_{\text {пр }}-m_{\Sigma}\left(\frac{k_{\text {пр }}}{m_{\Sigma}}-\left(\frac{R_{\text {мех }}}{m_{\Sigma}}\right)^{2}\right)\right]^{2}+\right. \\
& \left.+R_{\text {мex }}^{2}\left[\frac{k_{\text {пр }}}{m_{\Sigma}}-\left(\frac{R_{\text {Mex }}}{2 m_{\Sigma}}\right)^{2}\right]\right\}^{-1} \times \\
& \times\left\{R_{\text {мех }}^{2}\left[\frac{k_{\text {пр }}}{m_{\Sigma}}-\left(\frac{R_{\text {мех }}}{2 m_{\Sigma}}\right)^{2}\right]^{2}+\left[\frac{k_{\text {пр }}}{m_{\Sigma}}-\left(\frac{R_{\text {мех }}}{2 m_{\Sigma}}\right)^{2}\right] k_{\text {пр }}^{2}-\right. \\
& \left.-2\left[\frac{k_{\text {пр }}}{m_{\Sigma}}-\left(\frac{R_{\text {мex }}}{2 m_{\Sigma}}\right)^{2}\right]^{2} k_{\text {пр }} m_{\Sigma}+\left[\frac{k_{\text {пр }}}{m_{\Sigma}}-\left(\frac{R_{\text {мex }}}{2 m_{\Sigma}}\right)^{2}\right]^{3} m_{\Sigma}^{2}\right\}^{\frac{1}{2}} \text {. }
\end{aligned}
$$

Амплитуда виброскорости $A_{V}(\omega)$ на граничных околорезонансных частотах будет определяться на основе следующего нелинейного алгебраического уравнения

$$
\frac{A_{V}\left(\omega_{0}\right)-A_{V}(0)}{2}=\underset{\omega \rightarrow \omega_{V \pi \_11}}{A_{V}(\omega)}=\underset{\omega \rightarrow \omega_{V} \pi_{-22}}{A} .
$$

Исходя из (6), составим равенство

$$
\begin{aligned}
& \frac{\left(R_{\text {мex }}^{2} \omega^{4}+\omega^{2} k_{\text {пр }}^{2}-2 \omega^{4} k_{\text {пp }} m_{\Sigma}+\omega^{6} m_{\Sigma}^{2}\right)^{\frac{1}{2}}}{\left(k_{\text {пр }}-m_{\Sigma} \grave{\mathrm{u}}^{2}\right)^{2}+R_{\text {Mex }}^{2} \grave{u ̀}^{2}}= \\
& =\frac{1}{2} \frac{1}{\left[\begin{array}{l}
{\left[k_{\text {пр }}-m_{\Sigma}\left(\frac{k_{\text {пр }}}{m_{\Sigma}}-\left(\frac{R_{\text {мех }}}{2 m_{\Sigma}}\right)^{2}\right)\right]^{2}+} \\
+R_{\text {мех }}^{2}\left(\frac{k_{\text {пр }}}{m_{\Sigma}}-\left(\frac{R_{\text {мех }}}{2 m_{\Sigma}}\right)^{2}\right)
\end{array}\right.} \times
\end{aligned}
$$

$$
\times\left\{R_{\text {мex }}^{2}\left(\frac{k_{\text {пр }}}{m_{\Sigma}}-\left(\frac{R_{\text {мex }}}{2 m_{\Sigma}}\right)^{2}\right)^{2}+\left(\frac{k_{\text {пр }}}{m_{\Sigma}}-\left(\frac{R_{\text {мex }}}{2 m_{\Sigma}}\right)^{2}\right) k_{\text {пр }}^{2}-\right.
$$$$
\left.-2\left[\frac{k_{\text {пр }}}{m_{\Sigma}}-\left(\frac{R_{\text {мex }}}{2 m_{\Sigma}}\right)^{2}\right]^{2} k_{\text {пр }} m_{\Sigma}+\left[\frac{k_{\text {пр }}}{m_{\Sigma}}-\left(\frac{R_{\text {мex }}}{2 m_{\Sigma}}\right)^{2}\right]^{3} m_{\Sigma}^{2}\right\}^{\frac{1}{2}} .
$$

а после преобразования получим уравнение

$$
\begin{aligned}
& \left(R_{\text {мех }}^{2} \omega^{4}+\omega^{2} k_{\text {пр }}^{2}-2 \omega^{4} k_{\text {пр }} m_{\Sigma}+\omega^{6} m_{\Sigma}^{2}\right)^{\frac{1}{2}}- \\
& -\left[\left(k_{\text {пр }}-m_{\Sigma} \omega^{2}\right)^{2}+R_{\text {мех }}^{2} \omega^{2}\right] \times \\
& \times \frac{1}{2} \frac{1}{\left[\left[k_{\text {пр }}-m_{\Sigma}\left(\frac{k_{\text {пр }}}{m_{\Sigma}}-\left(\frac{R_{\text {мех }}}{2 m_{\Sigma}}\right)^{2}\right)\right]^{2}+\right.} \times \\
& \times\left\{R_{\text {мех }}^{2}\left(\frac{k_{\text {пр }}}{m_{\Sigma}}-\left(\frac{R_{\text {мех }}}{2 m_{\Sigma}}\right)^{2}\right)^{2}+\left(\frac{k_{\text {пр }}}{m_{\Sigma}}-\left(\frac{R_{\text {мех }}}{2 m_{\Sigma}}\right)^{2}\right) k_{\text {пр }}^{2}-\right. \\
& -2\left[\frac{k_{\text {пр }}}{m_{\Sigma}}-\left(\frac{R_{\text {мех }}}{2 m_{\Sigma}}\right)^{2}\right]^{2} k_{\text {пр }} m_{\Sigma}+ \\
& \left.+\left[\frac{k_{\text {пр }}}{m_{\Sigma}}-\left(\frac{R_{\text {мех }}}{2 m_{\Sigma}}\right)^{2}\right]^{3} m_{\Sigma}^{2}\right\}^{\frac{1}{2}}=0
\end{aligned}
$$

Получено уравнение восьмого порядка, решив которое найдём восемь корней. Произведя процедуру отбора корней уравнения (7), получим два искомых корня уравнения. Меньшим из корней является нижняя граничная частота полосы пропускания скоростной амплитудно-частотной характеристики (рис. 9, 10) 

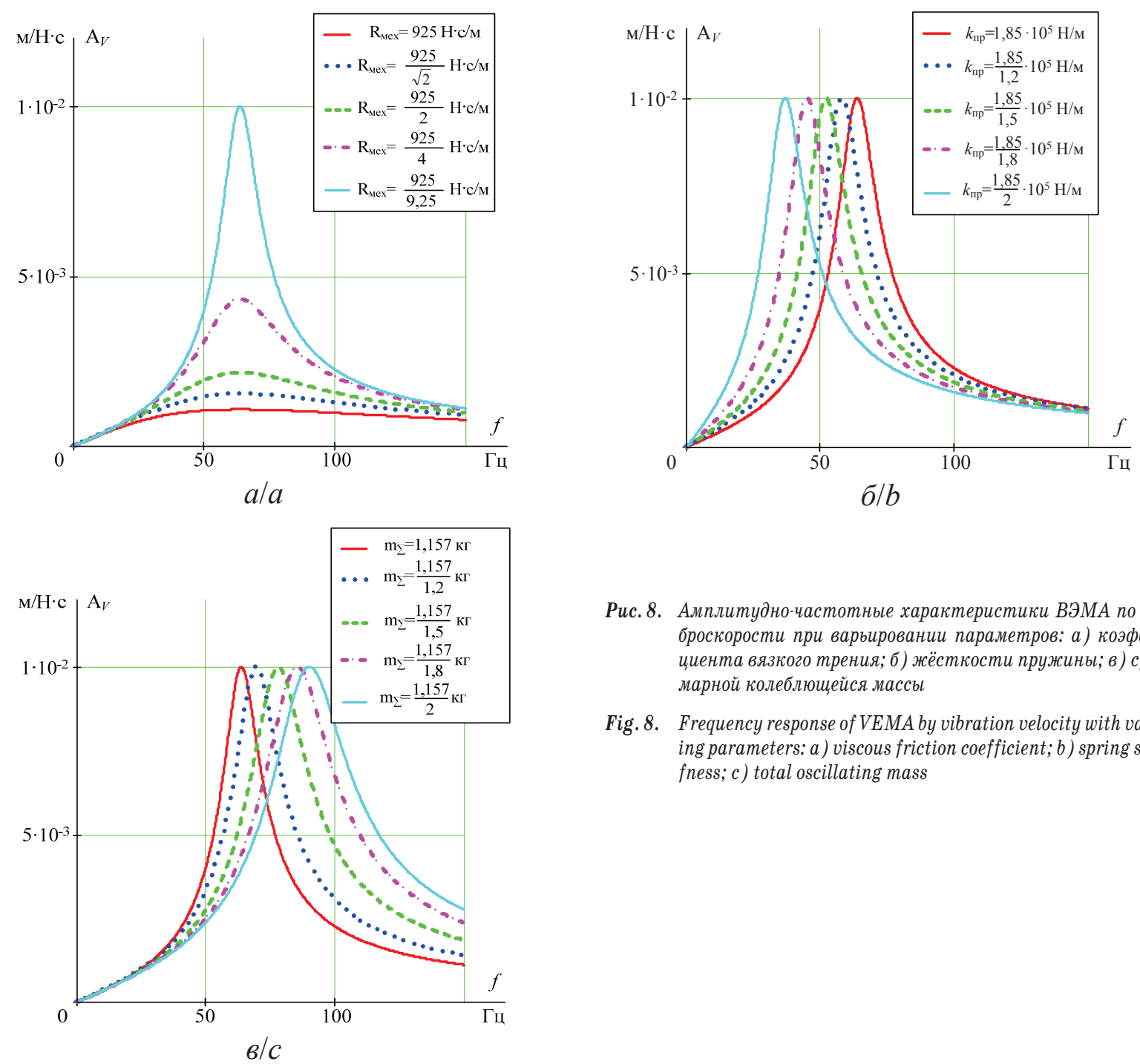

Pис. 8. Амплитудно-частотные характеристики ВЭМА по виброскорости при варьировании параметров: а) коэффиииента вязкого трения; б) жёсткости пружины; в) суммарной колеблющейся массы

Fig. 8. Frequency response of VEMA by vibration velocity with varying parameters: a) viscous friction coefficient; $b$ ) spring stiffness; c) total oscillating mass
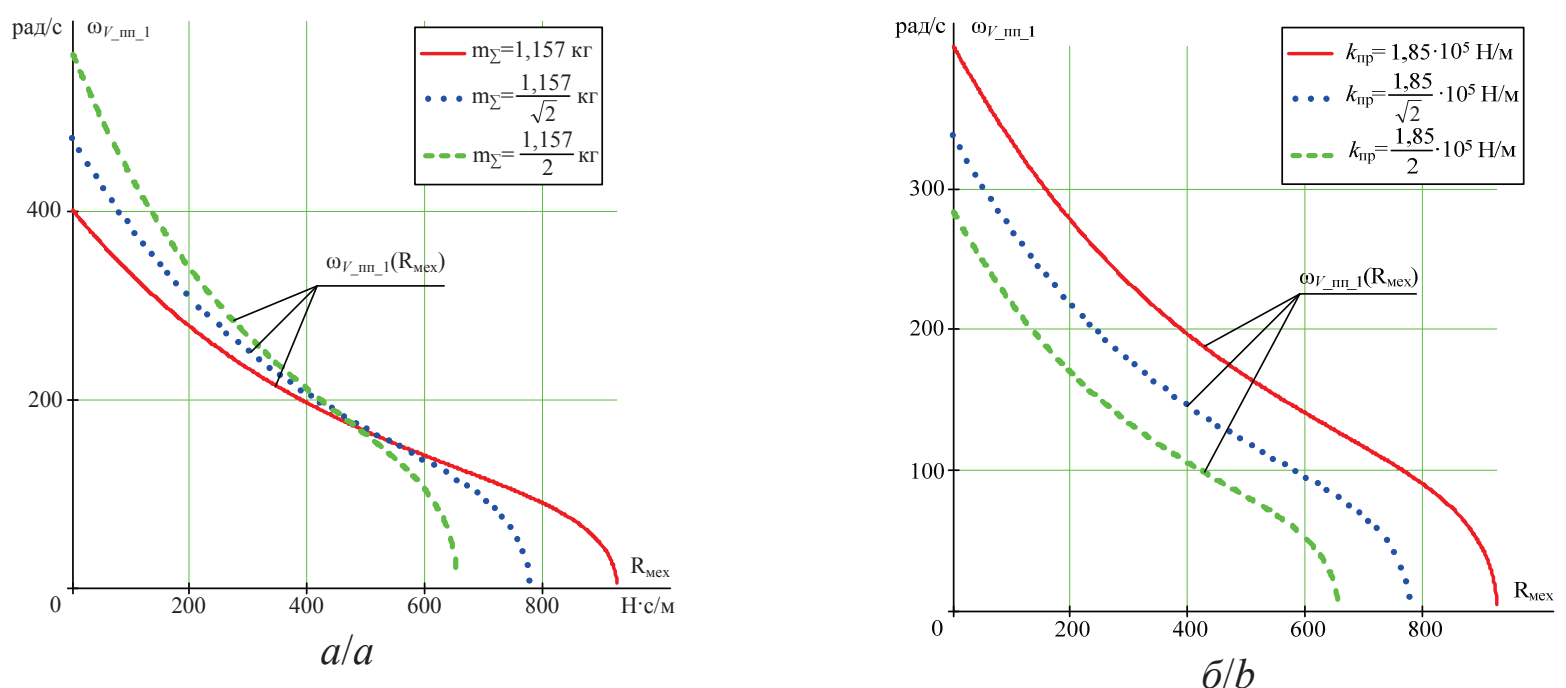

Pис. 9. Зависимость нижней граничной частоты полосы пропускания АЧХ ВЭМА по виброскорости от коэффициента вязкого трения при варьировании параметров: а) сумларной колеблющейся массы; б) жёсткости пружины.

Fig. 9. Dependence of the lower limiting frequency of the VEMA frequency response of by vibration velocity on the viscous friction coefficient with varying parameters: a) total oscillating mass; $b$ ) spring stiffness 

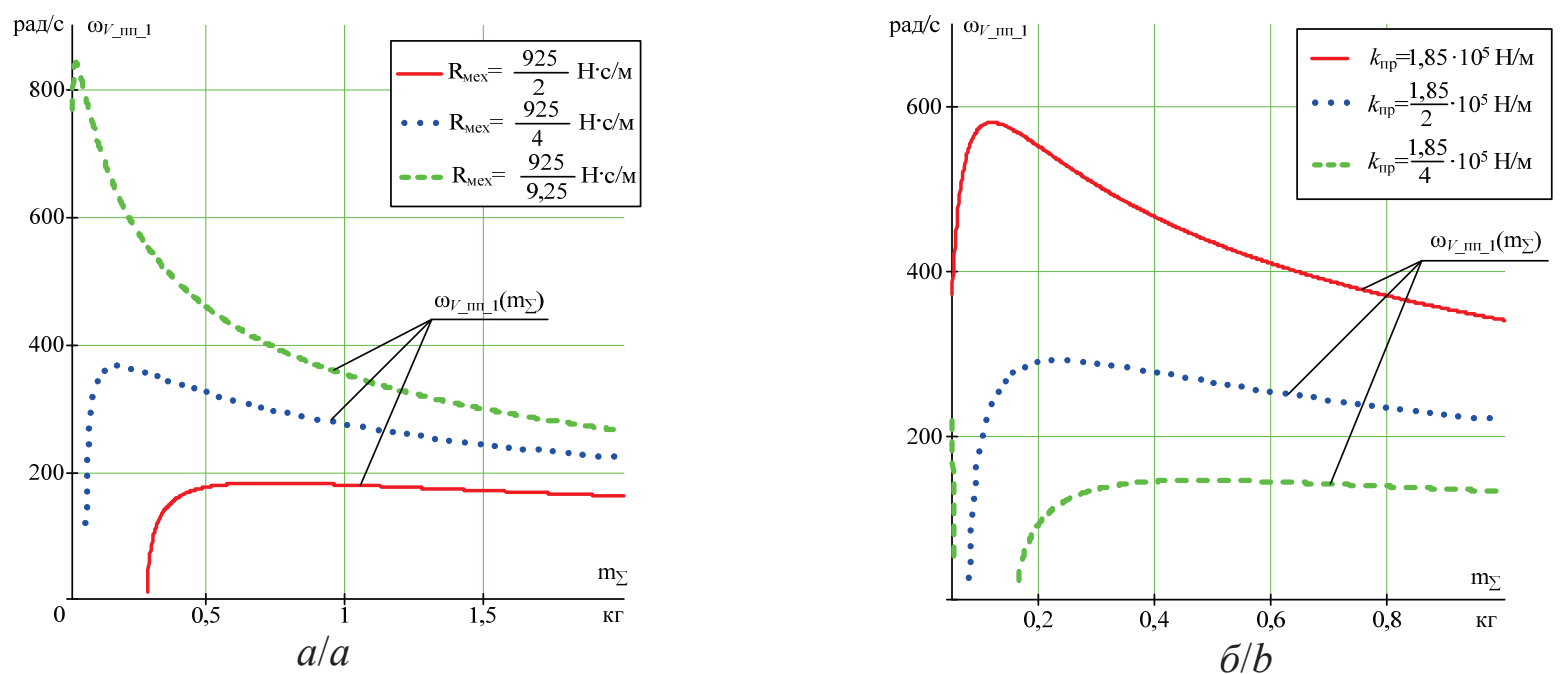

Рис. 10. Зависимость нижней граничной частоты полосы пропускания АЧХ ВЭМА по виброскорости от суммарной колеблющейся массы при варьировании параметров: а) коэффиииента вязкого трения; б) жёсткости пружины

Fig. 10. Dependence of the lower limiting frequency of the VEMA frequency response of by vibration velocity on the total oscillating mass with varying parameters: a) total viscous friction coefficient; $b$ ) spring stiffness
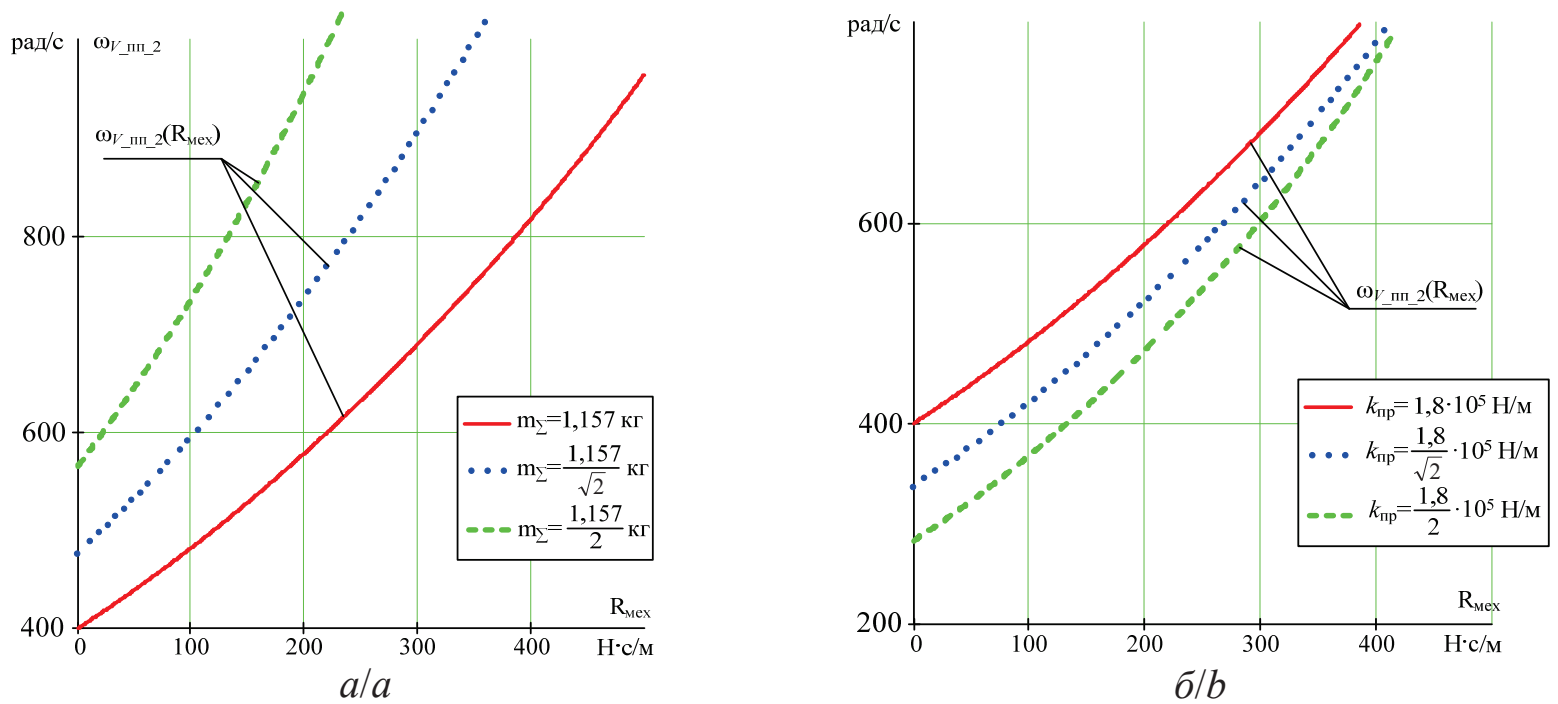

Рис. 11. Зависимость верхней граничной частоты полосы пропускания АЧХ ВЭМА по виброскорости от коэффициента вязкого трения при варьировании параметров: а) суммарной колеблющейся массы; б) жёсткости пружины

Fig. 11. Dependence of the higher limiting frequency of the VEMA frequency response by vibration velocity on the viscous friction coefficient with varying parameters: a) total oscillating mass; $b$ ) spring stiffness

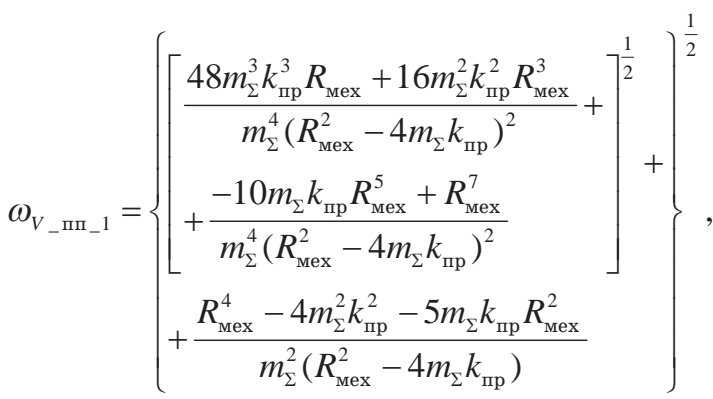

а большим из корней является верхняя граничная частота полосы пропускания скоростной амплитудно-частотной характеристики (рис. 11,12$)$

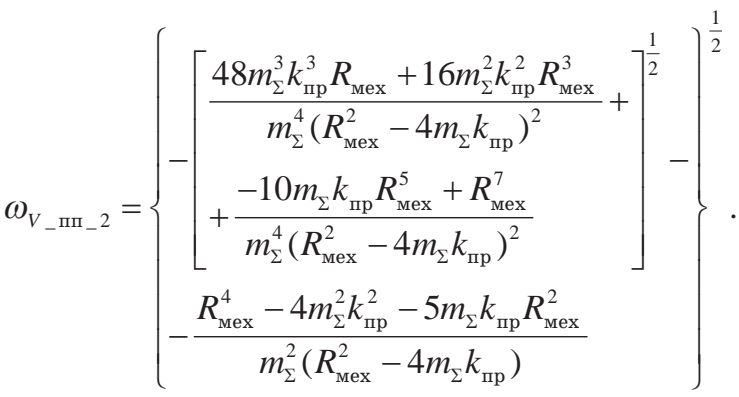

Имея частоты, ограничивающие полосу пропускания, выведем выражение для ширины полосы пропускания скоростной амплитудно-частотной характеристики (рис. 12, 13) 

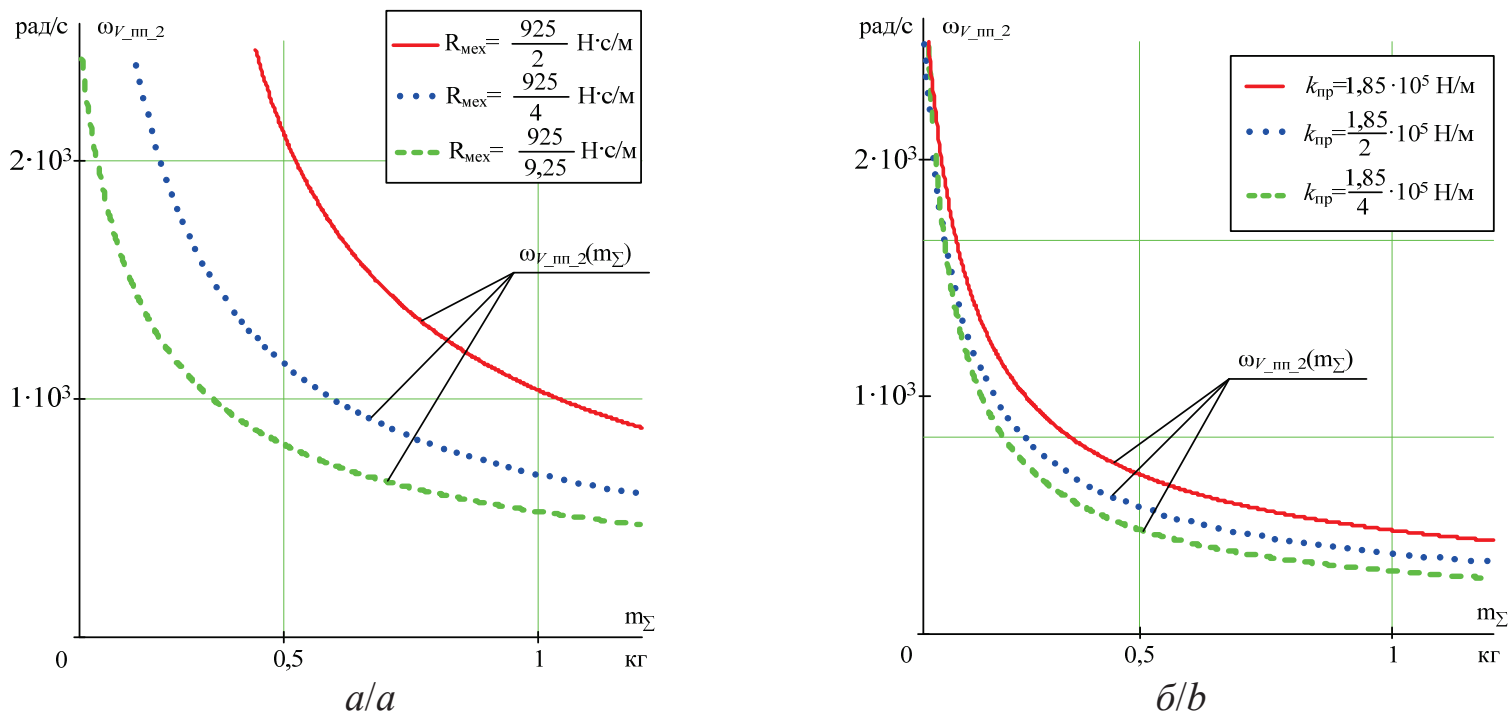

Рис. 12. Зависимость верхней граничной частоты полосы пропускания АЧХ ВЭМА по виброскорости от сумларной колеблющейся массы при варьировании параметров: а) коэффициента вязкого трения; б) жёсткости пружины.

Fig. 12. Dependence of the higher limiting frequency of the VEMA frequency response by vibration velocity on the total oscillating mass with varying parameters: a) total viscous friction coefficient; $b$ ) spring stiffness
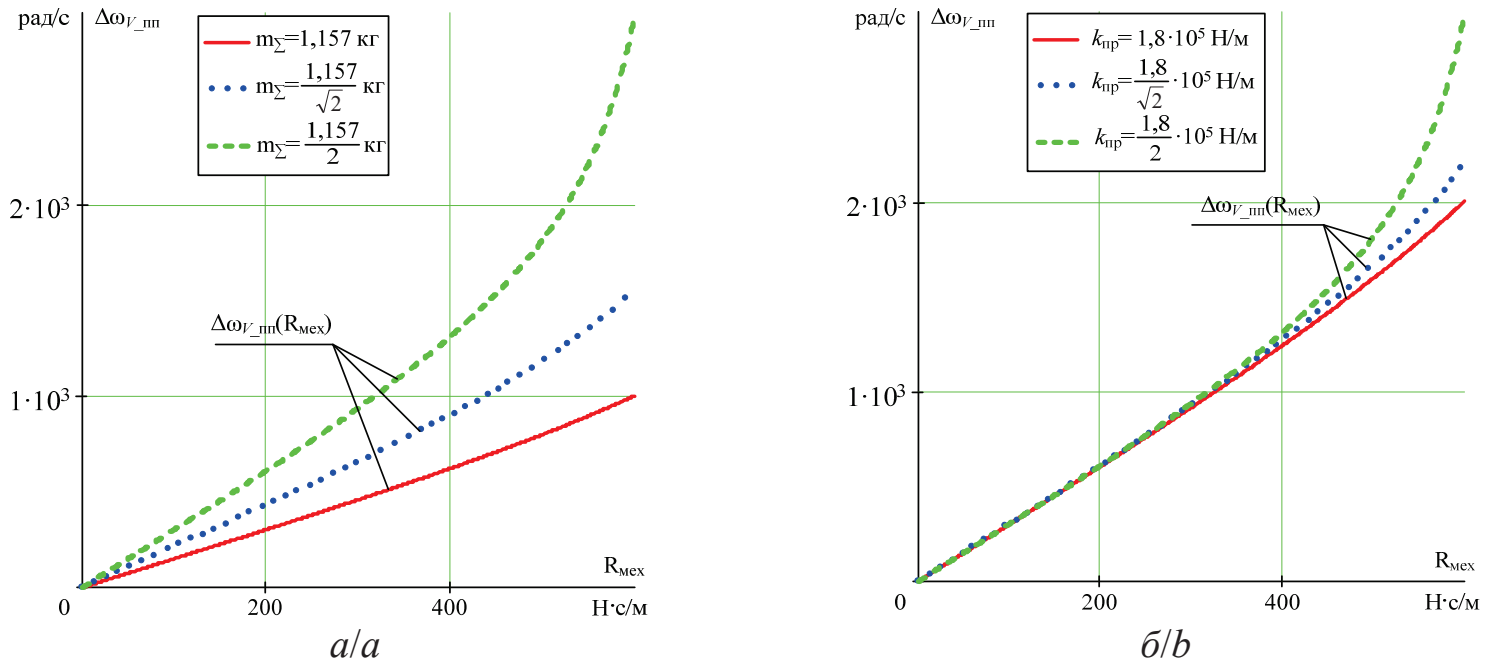

Рис. 13. Зависимость ширины полосы пропускания АЧХ ВЭМА по виброскорости от коэффициента вязкого трения при варьировании параметров: а) суммарной колеблющейся массы; б) жёсткости пружины

Fig. 13. Dependence of the bandwidth of the VEMA frequency response by vibration velocity on the viscous friction coefficient with varying parameters: a) total oscillating mass; $b$ ) spring stiffness

$$
\Delta \omega_{V_{-} \text {III }}=\omega_{V_{-} \text {III_21 }}-\omega_{V_{-} \text {III_ } 1} \text {. }
$$

В случае если для измерения параметров вибрации используется сигнал от датчика виброскорости, передаточная функция механического контура по ускорению - отношение величины виброускорения к приложенной к механическому контуру электромагнитной силе

$$
W_{\mathrm{a}}(p)=\frac{a(p)}{F_{\text {эм }}(p)}=\frac{p^{2}}{\left(m_{\Sigma} p^{2}+R_{\text {мех }} p+k_{\text {пр }}\right)} .
$$

Амплитуда ускорения $a(p)$ пропорциональна произведению $F_{\text {эм }}(p)$ на $W_{\text {а }}(p)$

$$
a(p)=\frac{F_{\text {эм }}(p) p^{2}}{\left(m_{\Sigma} p^{2}+R_{\text {мех }} p+k_{\text {пр }}\right)}=F_{\text {эм }}(p) W_{\mathrm{a}}(p) .
$$

После подстановки $p=j \omega$ получим $W_{\text {a }}(j \omega)$ и представим её составляющими $P_{\mathrm{a}}(\omega)$ и $Q_{\mathrm{a}}(\omega)$

$$
\begin{gathered}
W_{\mathrm{a}}(j \omega)=\frac{(j \omega)^{2}}{\left(m_{\Sigma}(j \omega)^{2}+R_{\text {мех }} j \omega+k_{\text {пр }}\right)}= \\
=\frac{-\omega^{2}}{\left(k_{\text {пр }}-m_{\Sigma} \omega^{2}\right)+R_{\text {мех }} j \omega} ; \\
W_{\mathrm{a}}(j \omega)=\frac{-\omega^{2}\left[\left(k_{\text {пр }}-m_{\Sigma} \omega^{2}\right)+R_{\text {мех }} j \omega\right]}{\left(k_{\text {пр }}-m_{\Sigma} \omega^{2}\right)+R_{\text {мех }} j \omega} \times \\
\times\left[\left(k_{\text {пр }}-m_{\Sigma} \omega^{2}\right)+R_{\text {мех }} j \omega\right]^{-1} ; \\
W_{\text {а }}(j \omega)=\frac{-\omega^{2}\left(\left(k_{\text {пр }}-m_{\Sigma} \omega^{2}\right)+R_{\text {мех }} j \omega\right)}{\left(k_{\text {пр }}-m_{\Sigma} \omega^{2}\right)^{2}+R_{\text {мех }}^{2} \omega^{2}} .
\end{gathered}
$$



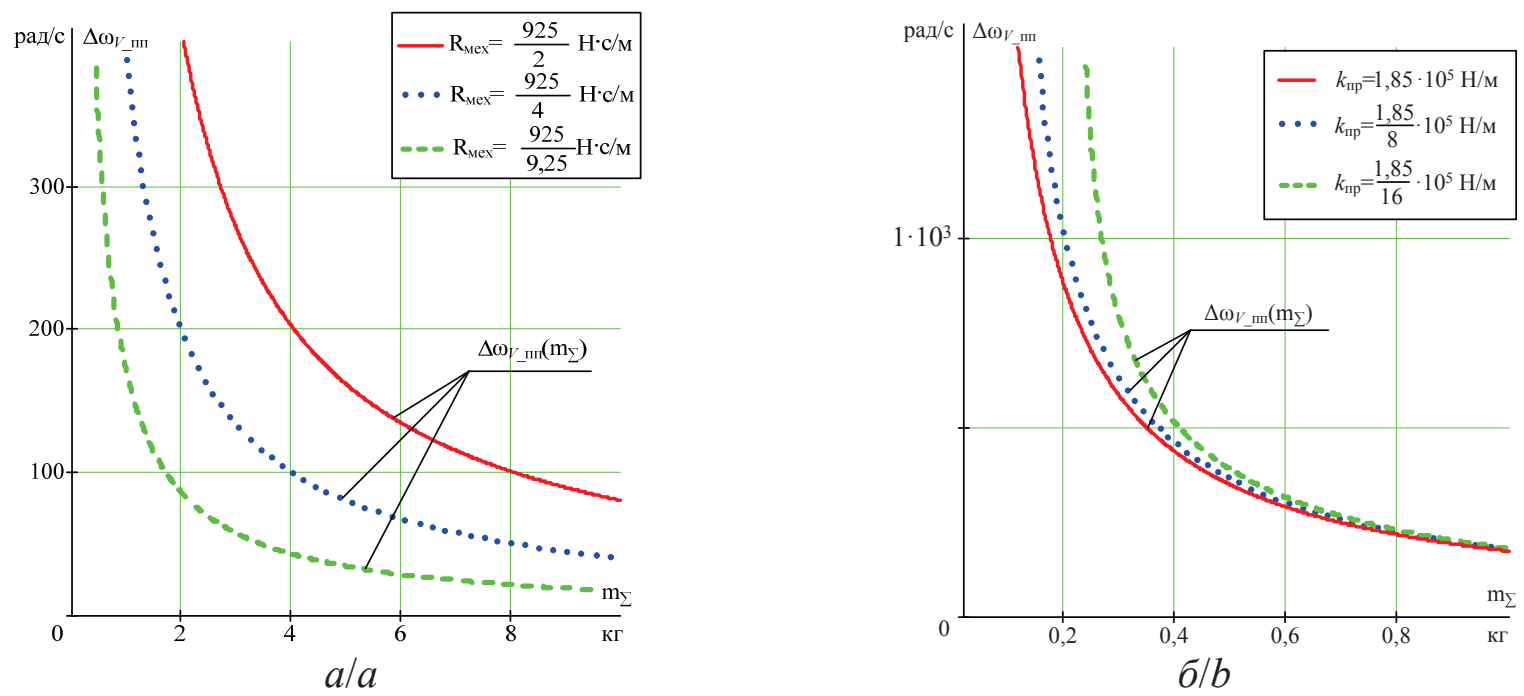

Рис. 14. Зависимость ширины полосы пропускания АЧХ ВЭМА по виброскорости от суммарной колеблющейся массы при варьировании параметров: а) коэффищиента вязкого трения; б) жёсткости пружины

Fig. 14. Dependence of the bandwidth of the vibration velocity of the VEMA frequency response on the total oscillating mass with varying parameters: a) total viscous friction coefficient; $b$ ) spring stiffness
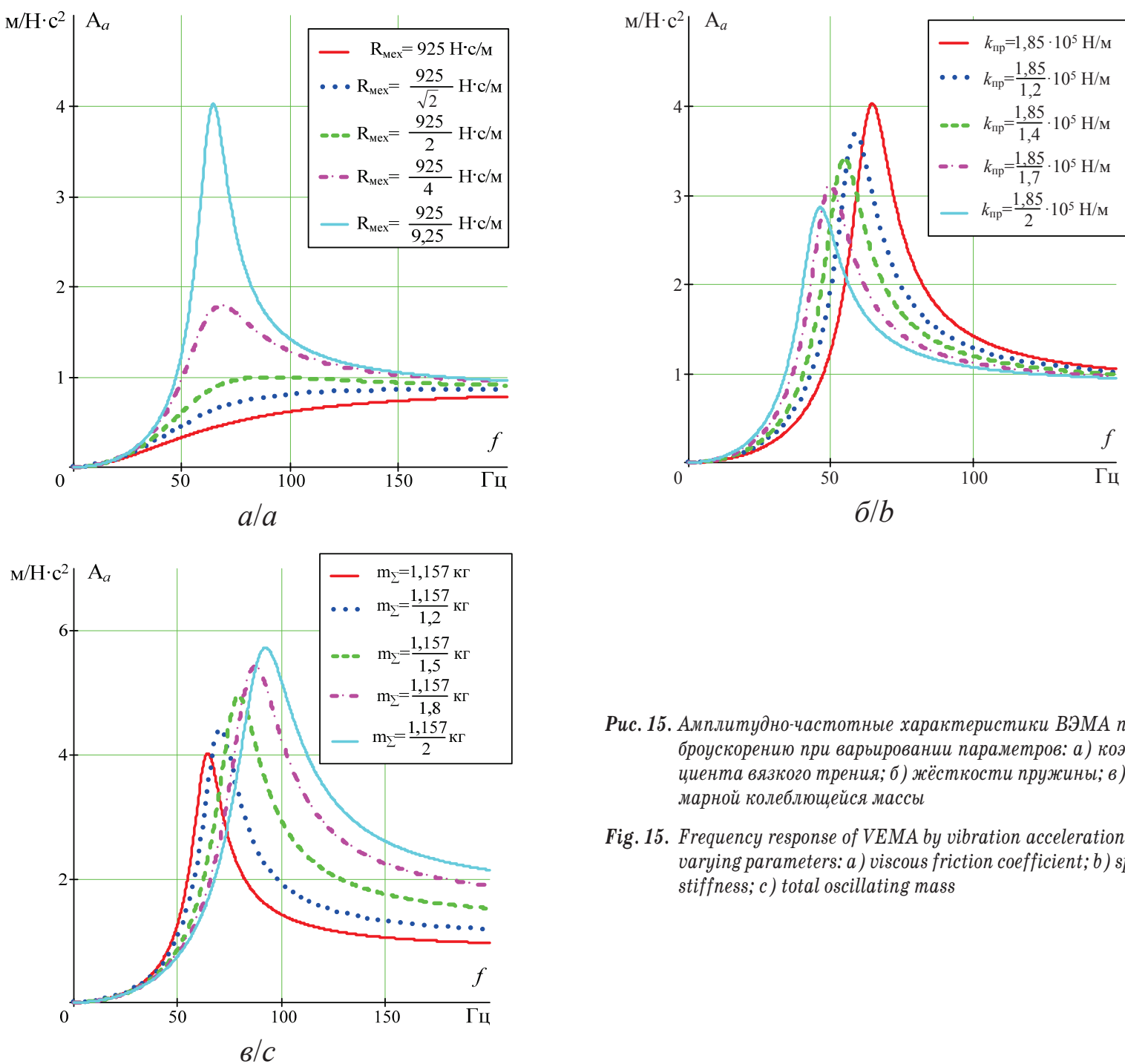

Рис. 15. Амплитудно-частотные характеристики ВЭМА по виброускорению при варьировании параметров: а) коэффициента вязкого трения; б) жёсткости пружины; в) суммарной колеблющейся массы

Fig. 15. Frequency response of VEMA by vibration acceleration with varying parameters: a) viscous friction coefficient; $b$ ) spring stiffness; c) total oscillating mass 
Комплексно-частотная характеристика по ускорению

$$
\begin{gathered}
W_{\mathrm{a}}(j \omega)=\frac{R_{\text {мex }} j \omega^{3}-\omega^{2}\left(k_{\text {пр }}-m_{\Sigma} \omega^{2}\right)}{\left(k_{\text {пр }}-m_{\Sigma} \omega^{2}\right)^{2}+R_{\text {мex }}^{2} \omega^{2}} ; \\
W_{\mathrm{a}}(j \omega)=\frac{-\omega^{2}\left(k_{\text {пр }}-m_{\Sigma} \omega^{2}\right)}{\left(k_{\text {пр }}-m_{\Sigma} \omega^{2}\right)^{2}+R_{\text {mex }}^{2} \omega^{2}}+ \\
+\frac{R_{\text {мex }} j \omega^{3}}{\left(k_{\text {пр }}-m_{\Sigma} \omega^{2}\right)^{2}+R_{\text {мex }}^{2} \omega^{2}} .
\end{gathered}
$$

Вещественная частотная характеристика по ускорению

$$
P_{\mathrm{a}}(\omega)=\frac{-\omega^{2}\left(k_{\text {пр }}-m_{\Sigma} \omega^{2}\right)}{\left(k_{\text {пр }}-m_{\Sigma} \omega^{2}\right)^{2}+R_{\text {мex }}^{2} \omega^{2}} .
$$

Мнимая частотная характеристика по ускорению

$$
Q_{\mathrm{a}}(\omega)=\frac{R_{\text {mex }} \omega^{3}}{\left(k_{\text {mp }}-m_{\Sigma} \omega^{2}\right)^{2}+R_{\text {mex }}^{2} \omega^{2}} .
$$

Амплитудно-частотная характеристика по ускорению (рис. 15)

$$
\begin{gathered}
A_{\mathrm{a}}(\omega)=\left|W_{\mathrm{a}}(j \omega)\right|=\sqrt{P_{\mathrm{a}}(\omega)^{2}+Q_{\mathrm{a}}(\omega)^{2}}= \\
=\frac{\sqrt{\left(-\omega^{2}\left(k_{\text {пр }}-m_{\Sigma} \omega^{2}\right)\right)^{2}+\left(R_{\text {мех }} \omega^{3}\right)^{2}}}{\left(k_{\text {пр }}-m_{\Sigma} \omega^{2}\right)^{2}+R_{\text {мех }}^{2} \omega^{2}} .
\end{gathered}
$$

При $\omega \rightarrow \infty$ амплитуда виброускорения стремится к пределу

$$
\begin{gathered}
\lim _{\substack{\mathrm{u} \rightarrow \infty \\
A_{a}}}(\omega)=\frac{\sqrt{\left(-\infty^{2}\left(k_{\text {пр }}-m_{\Sigma} \infty^{2}\right)\right)^{2}+\left(R_{\text {мех }} \infty^{3}\right)^{2}}}{\left(k_{\text {пр }}-m_{\Sigma} \infty^{2}\right)^{2}+R_{\text {мex }}^{2} \infty^{2}} ; \\
\lim _{\substack{\omega \rightarrow \infty \\
A_{a}}}(\omega) \rightarrow \frac{1}{m_{\Sigma}} .
\end{gathered}
$$

Производная по частоте от амплитудно-частотной характеристики по ускорению

$$
\begin{gathered}
d A_{-} \omega_{V}(\omega)=\frac{d A_{a}(\omega)}{d \omega}=\frac{1}{\left(k_{\text {mp }}-m_{\Sigma} \omega^{2}\right)^{2}+R_{\text {mex }}^{2} \omega^{2}} \times \\
\times \frac{4 \omega^{3} k_{\text {mp }}^{2}-12 \omega^{5} k_{\text {mp }} m_{\Sigma}+8 \omega^{7} m_{\Sigma}^{2}+6 \omega^{5} R_{\text {mex }}^{2}}{2 \sqrt{\omega^{4} k_{\text {mp }}^{2}-2 \omega^{6} k_{\text {mp }} m_{\Sigma}+\omega^{8} m_{\Sigma}^{2}+\omega^{6} R_{\text {mex }}^{2}}}- \\
-\frac{\sqrt{\omega^{4} k_{\text {mp }}^{2}-2 \omega^{6} k_{\text {mp }} m_{\Sigma}+\omega^{8} m_{\Sigma}^{2}+\omega^{6} R_{\text {mex }}^{2}}}{\left[\left(k_{\text {mp }}-m_{\Sigma} \omega^{2}\right)^{2}+R_{\text {mex }}^{2} \omega^{2}\right]^{2}} \times \\
\times\left(-4\left(k_{\text {mp }}-m_{\Sigma} \omega^{2}\right) m_{\Sigma} \omega+2 R_{\text {mex }}^{2} \omega\right) .
\end{gathered}
$$

Приравняем (8) к нулю. Решим полученное уравнение. Получим два корня. Отрицательный отбросим. Резонансная частота для амплитудночастотной характеристики по ускорению (рис. 15)

$$
\omega_{a 0}=\frac{-k_{\mathrm{mp}}}{R_{\mathrm{mex}}^{2}-2 k_{\mathrm{mp}} m_{\Sigma}} \sqrt{4 k_{\mathrm{mp}} m_{\Sigma}-2 R_{\mathrm{mex}}^{2}} .
$$

При стремлении вынуждающей частоты к частоте свободных колебаний механического конту- ра амплитуда виброускорения аналогично стремится к максимуму, величина которого зависит от параметров механического контура

$$
\begin{gathered}
A_{\mathrm{a}}\left(\omega_{0}\right)=\left\{\begin{array}{l}
{\left[k_{\text {mp }}-\frac{m_{\Sigma} k_{\text {mp }}^{2}\left(4 m_{\Sigma} k_{\text {mp }}-2 R_{\text {mex }}^{2}\right)}{\left(-2 k_{\text {mp }} m_{\Sigma}+R_{\text {mex }}^{2}\right)^{2}}\right]^{2}+} \\
+\frac{R_{\text {mex }}^{2}\left(4 m_{\Sigma} k_{\text {mp }}-2 R_{\text {mex }}^{2}\right) k_{\text {mp }}^{2}}{\left(-2 k_{\text {mp }} m_{\Sigma}+R_{\text {mex }}^{2}\right)^{2}}
\end{array}\right]^{-1} \times \\
\times\left\{\begin{array}{l}
\frac{k_{\text {mp }}^{4}\left(4 m_{\Sigma} k_{\text {mp }}-2 R_{\text {mex }}^{2}\right)^{2}}{\left(-2 k_{\text {mp }} m_{\Sigma}+R_{\text {mex }}^{2}\right)^{4}} \times \\
\times\left[k_{\text {пр }}-\frac{m_{\Sigma} k_{\text {mp }}^{2}\left(4 m_{\Sigma} k_{\text {mp }}-2 R_{\text {mex }}^{2}\right)}{\left(-2 k_{\text {mp }} m_{\Sigma}+R_{\text {mex }}^{2}\right)^{2}}\right]^{2} \\
+\frac{\left(4 m_{\Sigma} k_{\text {mp }}-2 R_{\text {mex }}^{2}\right)^{3} k_{\text {mp }}^{6} R_{\text {mex }}^{2}}{\left(-2 k_{\text {mp }} m_{\Sigma}+R_{\text {mex }}^{2}\right)^{6}}
\end{array}\right.
\end{gathered}
$$

Найдём частоты, ограничивающие полосу пропускания. Зная, что

$$
\frac{A_{a}\left(\omega_{0}\right)-A_{a}(0)}{2}=\underset{\omega \rightarrow \omega_{a_{-} \mathrm{m} \_} 2}{A}=\underset{\omega \rightarrow \omega_{a_{-} \mathrm{mL} \_} 1}{A(\omega)},
$$

составим равенство

$$
\begin{aligned}
& \frac{1}{2}\left[\begin{array}{l}
\left(k_{\text {mp }}-\frac{m_{\Sigma} k_{\text {mp }}^{2}\left(4 m_{\Sigma} k_{\text {mp }}-2 R_{\text {mex }}^{2}\right)}{\left(-2 k_{\text {mp }} m_{\Sigma}+R_{\text {mex }}^{2}\right)^{2}}\right)^{2}+ \\
+\frac{R_{\text {mex }}^{2}\left(4 m_{\Sigma} k_{\text {mp }}-2 R_{\text {mex }}^{2}\right) k_{\text {mp }}^{2}}{\left(-2 k_{\text {mp }} m_{\Sigma}+R_{\text {mex }}^{2}\right)^{2}}
\end{array}\right]^{-1} \times \\
& \times\left[\begin{array}{l}
\frac{k_{\text {mp }}^{4}\left(4 m_{\Sigma} k_{\text {mp }}-2 R_{\text {mex }}^{2}\right)^{2}}{\left(-2 k_{\text {mp }} m_{\Sigma}+R_{\text {mex }}^{2}\right)^{4}} \times \\
\times\left(k_{\text {mp }}-\frac{m_{\Sigma} k_{\text {mp }}^{2}\left(4 m_{\Sigma} k_{\text {mp }}-2 R_{\text {mex }}^{2}\right)}{\left(-2 k_{\text {mp }} m_{\Sigma}+R_{\text {mex }}^{2}\right)^{2}}\right)^{2}+ \\
+\frac{\left(4 m_{\Sigma} k_{\text {mp }}-2 R_{\text {mex }}^{2}\right)^{3} k_{\text {mp }}^{6} R_{\text {mex }}^{2}}{\left(-2 k_{\text {mp }} m_{\Sigma}+R_{\text {mex }}^{2}\right)^{6}}
\end{array}\right]^{\frac{1}{2}}= \\
& =\frac{\sqrt{\left(-\omega^{2}\left(k_{\text {mp }}-m_{\Sigma} \omega^{2}\right)\right)^{2}+\left(R_{\text {mex }} \omega^{3}\right)^{2}}}{\left(k_{\text {mp }}-m_{\Sigma} \omega^{2}\right)^{2}+R_{\text {mex }}^{2} \omega^{2}} .
\end{aligned}
$$

Решим это уравнение и после процедуры отбора корней определим границы полосы пропускания для амплитудно-частотной характеристики по ускорению. Нижняя граничная частота полосы пропускания для амплитудно-частотной характеристики по ускорению (рис. 16,17 )

$$
\omega_{a_{-} \text {III_ } 1}=\left[\begin{array}{c}
\frac{R_{\text {mex }}^{2} k_{\text {mp }}^{2}-2 m_{\Sigma} k_{\text {mp }}^{3}}{-2\left(m_{\Sigma}^{2} k_{\text {mp }}^{2}-4 m_{\Sigma} k_{\text {mp }} R_{\text {mex }}^{2}+R_{\text {mex }}^{4}\right)}+ \\
+\frac{\sqrt{3} k_{\text {mp }}^{2} R_{\text {mex }} \sqrt{4 m_{\Sigma} k_{\text {mp }}-R_{\text {mex }}^{2}}}{2\left(m_{\Sigma}^{2} k_{\text {mp }}^{2}-4 m_{\Sigma} k_{\text {mp }} R_{\text {mex }}^{2}+R_{\text {mex }}^{4}\right)}
\end{array}\right]^{\frac{1}{2}} .
$$



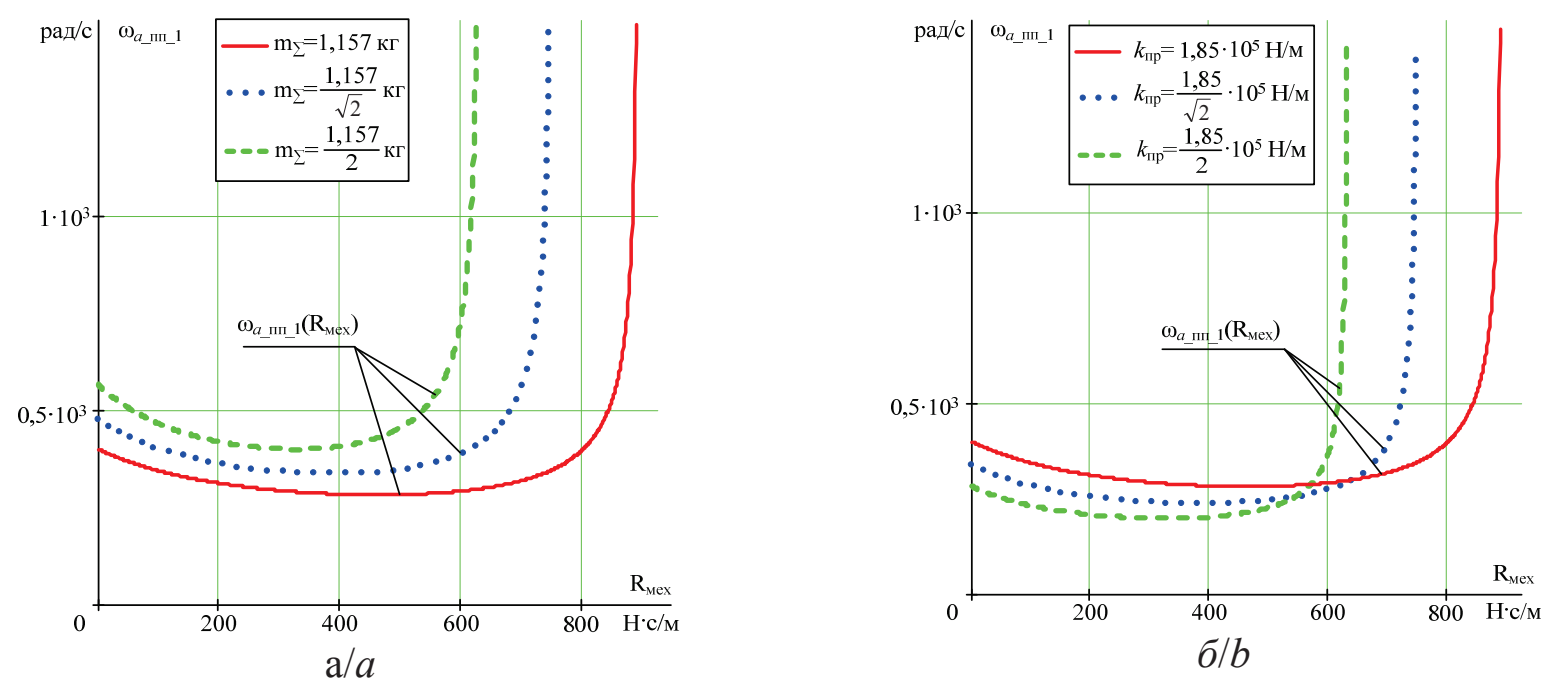

Рис. 16. Зависимость нижней граничной частоты полосы пропускания АЧХ ВЭМА по виброускорению от коэффициента вязкого трения при варьировании параметров: а) суммарной колеблющейся массы; б) жёсткости пружины

Fig. 16. Dependence of the lower limiting frequency of the VEMA frequency response by vibration acceleration on the viscous friction coefficient with varying parameters: a) total oscillating mass; $b$ ) spring stiffness
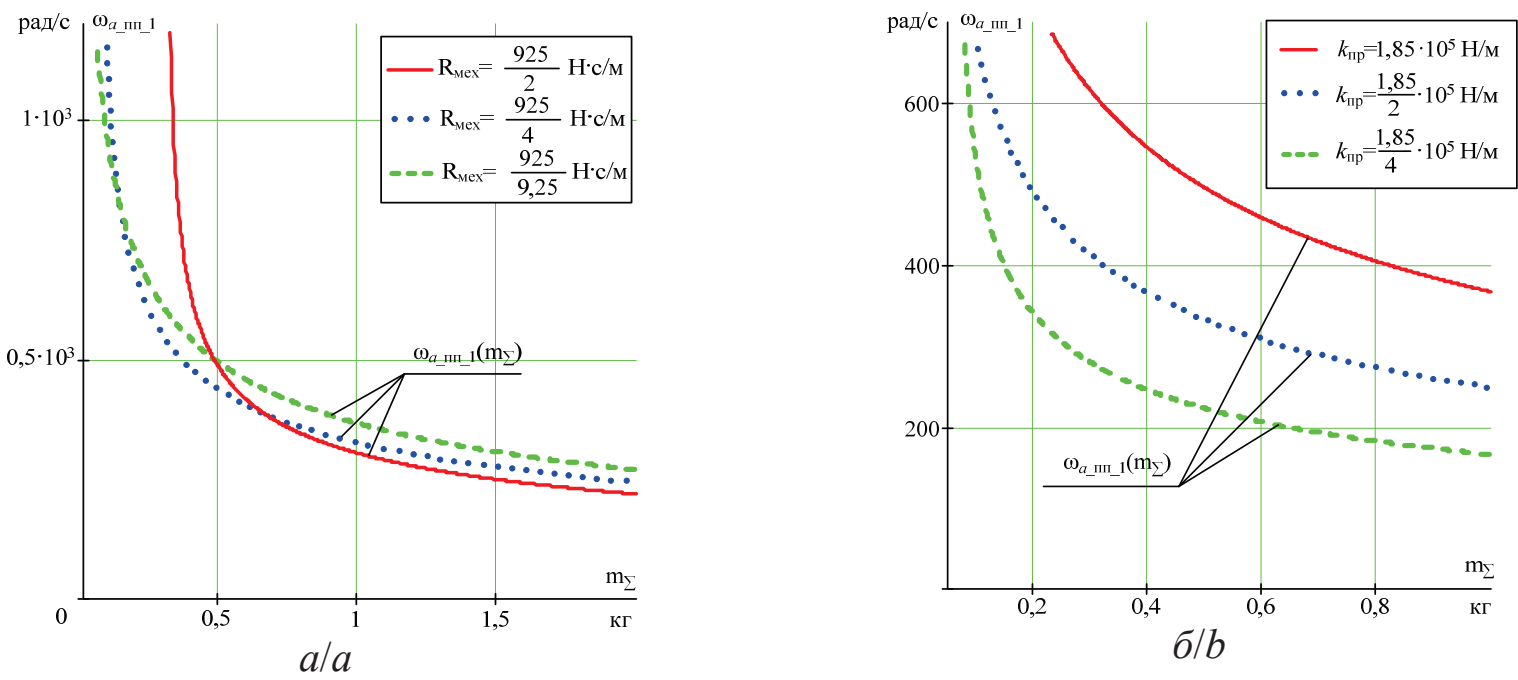

Рис. 17. Зависимость нижней граничной частоты полосы пропускания АЧХ ВЭМА по виброускорению от суммарной колеблющейся массы при варьировании параметров: а) коэффиииента вязкого трения; б) жёсткости пружины

Fig. 17. Dependence of the lower limiting frequency of the VEMA frequency response by vibration acceleration on the total oscillating mass with varying parameters: a) total viscous friction coefficient; $b$ ) spring stiffness

Верхняя граничная частота полосы пропускания для амплитудно-частотной характеристики по ускорению (рис. 18, 19)

$$
\omega_{a_{-} \text {пाI } \_}=\left[\begin{array}{c}
\frac{m_{\Sigma} k_{\text {mp }}^{3}-\frac{k_{\text {mp }}^{2} R_{\text {mex }}^{2}}{2}}{m_{\Sigma}^{2} k_{\text {mp }}^{2}-4 m_{\Sigma} k_{\text {mp }} R_{\text {mex }}^{2}+R_{\text {mex }}^{4}}+ \\
\frac{\sqrt{3} k_{\text {mp }}^{2} R_{\text {mex }} \sqrt{4 m_{\Sigma} k_{\text {mp }}-R_{\text {mex }}^{2}}}{2} \\
+\frac{2}{m_{\Sigma}^{2} k_{\text {mp }}^{2}-4 m_{\Sigma} k_{\text {mp }} R_{\text {mex }}^{2}+R_{\text {mex }}^{4}}
\end{array}\right]^{\frac{1}{2}} .
$$

Имея частоты, ограничивающие полосу пропускания, выведем выражение для ширины полосы пропускания амплитудно-частотной характеристики по ускорению (рис. 20, 21)

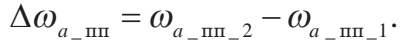

\section{Исследование границ применимости метода идентификация параметров механической системы вибрационного электромагнитного активатора по граничным околорезонанстным частотам}

Как видно из рис. 1 , амплитудно-частотные характеристики ВЭМА по вибросмещению при относительно небольших значениях коэффициента вязкого трения $R_{\text {мех }}$ имеют явно выраженные максимумы, а при стремлении $R_{\text {мех }}$ к граничному зна- 

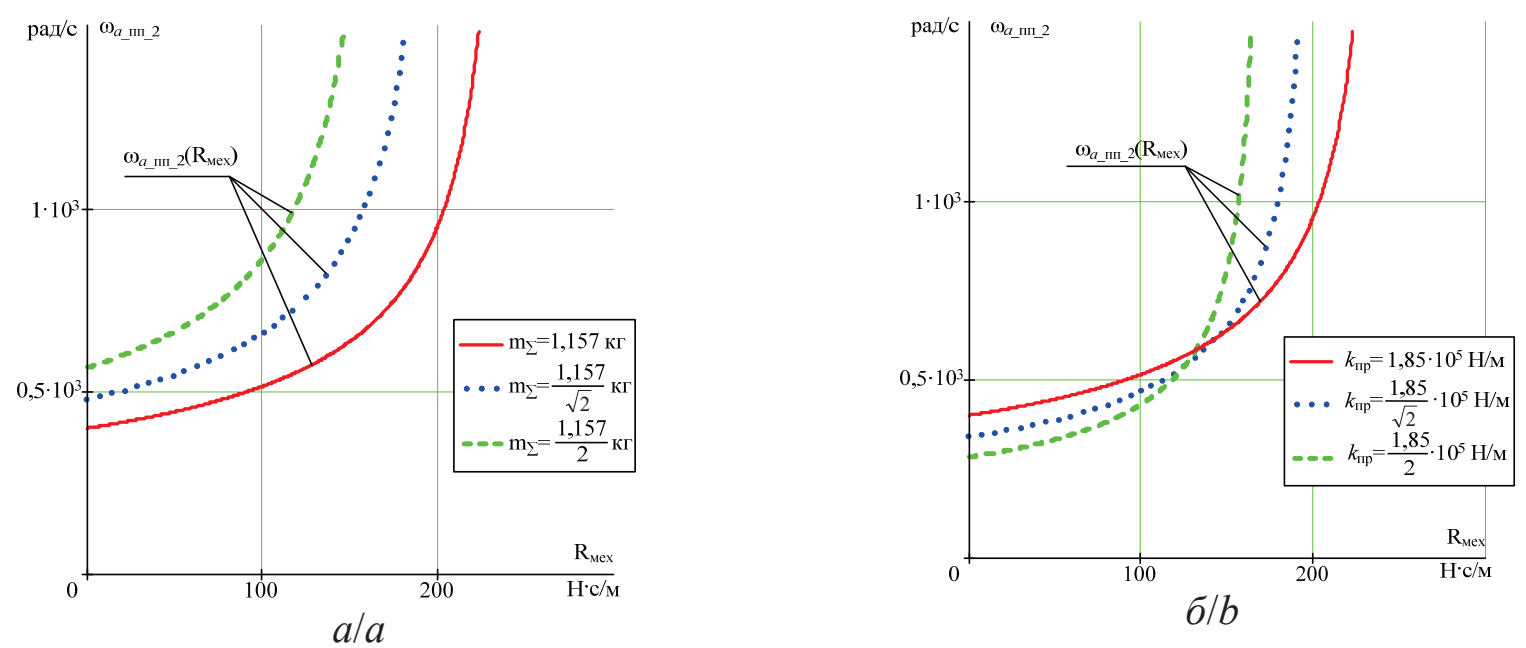

Рис. 18. Зависимость верхней граничной частоты полосы пропускания АЧХ ВЭМА по виброускорению от коэффициента вязкого трения при варьировании параметров: а) суммарной колеблющейся массы; б) жёсткости пружины.

Fig. 18. Dependence of the higher limiting frequency of the frequency response of VEMA by vibration acceleration from the viscous friction coefficient with varying parameters a) total oscillating mass b) spring stiffness
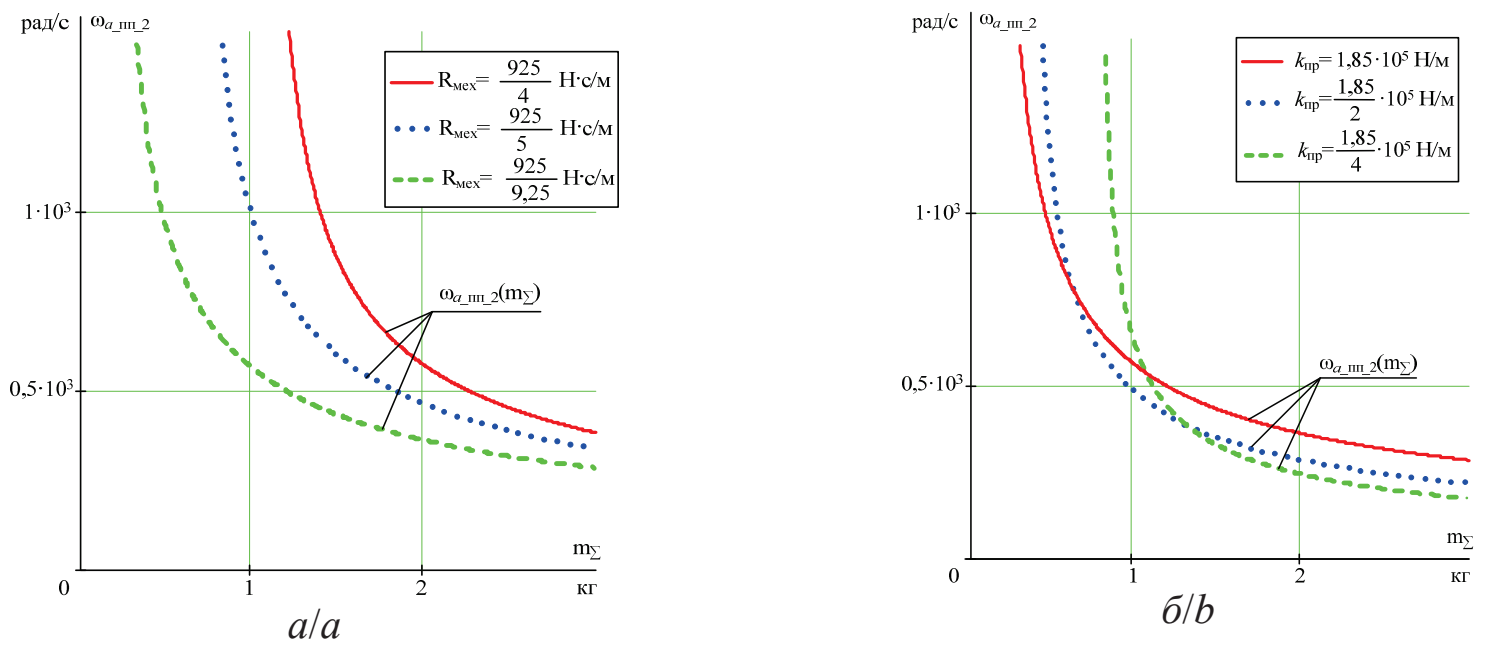

Рис. 19. Зависимость верхней граничной частоты полосы пропускания АЧХ ВЭМА по виброускорению от суммарной колеблющейся массы при варьировании параметров: а) коэффициента вязкого трения; б) жёсткости пружины

Fig. 19. Dependence of the higher limiting frequency of the VEMA frequency response by vibration acceleration on the total oscillating mass with varying parameters: a) total viscous friction coefficient; $b$ ) spring stiffness

чению $R_{\text {мех }} \rightarrow R_{\text {мех гр }}=2 \sqrt{ } k_{\text {пр }} m_{\Sigma}$ резонансный пик становится более сглаженным. Очевидно, что существует некоторое предельное значение коэффициента вязкого трения $R_{\text {мех.пред }}\left(m_{\Sigma .6}, k_{\text {пр.б}}\right)<R_{\text {мехггр }}$, при котором процесс вывода энергии из механического колебательного контура, т. е. демпфирование, преобладает над процессами взаимообмена энергии между пружинной с жесткостью $k_{\text {пр. }}$ и суммарной присоединенной массы $m_{\Sigma .6}$, где для известной конструкции ВЭМА-0,3 [11] за базовую величину жесткости пружины возьмем $k_{\text {пр. }}=1,85 \cdot 10^{5} \mathrm{H} / \mathrm{m}$, a за базовую величину суммарной присоединенной массы возьмем $m_{\Sigma \sigma}=1,157 \mathrm{\kappa}$.

Если условие $R_{\text {мех.пред }}\left(m_{\Sigma .5}, k_{\text {пр.б}}\right)<R_{\text {мех_гр }}$ не выполняется, то при попытке применения предложенного метода исследователь столкнется с тем, что результаты решения алгебраического уравнения (3) являются комплексными корнями и применять описанный метод становится невозможным.

Исследование границ применимости метода идентификации параметров механической системы вибрационного электромагнитного активатора по граничным околорезонанстным частотам проведем на основе амплитудно-частотных характеристик по виброперемещению. Методика исследования границ применимости метода на основе АЧХ по виброскорости и виброускорению подобна описанной и в статье не приводится.

На основе вышеописанной математической модели механического контура ВЭМА найдены соотношения между предельными значениями коэффициента вязкого трения $R_{\text {мех.пред }}$, жесткости пружины $k_{\text {пр.б }}$ и суммарной присоединенной массы $m_{\Sigma .6}$ (таблица). 

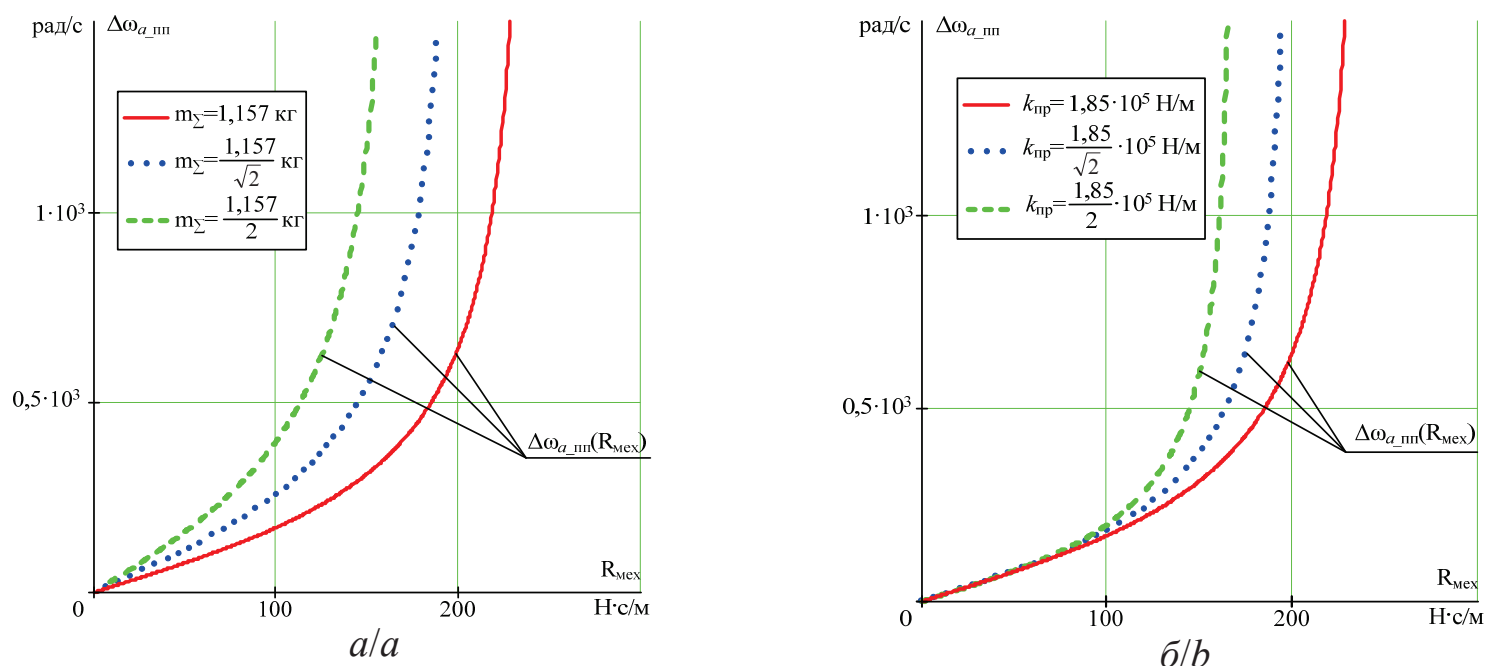

Рис. 20. Зависимость ширины полосы пропускания АЧХ ВЭМА по виброускорению от коэффициента вязкого трения при варьировании параметров: а) суммарной колеблющейся массы; б) жёсткости пружины

Fig. 20. Dependence of the bandwidth of the VEMA frequency response by vibration acceleration on the viscous friction coefficient with varying parameters: a) total oscillating mass; $b$ ) spring stiffness
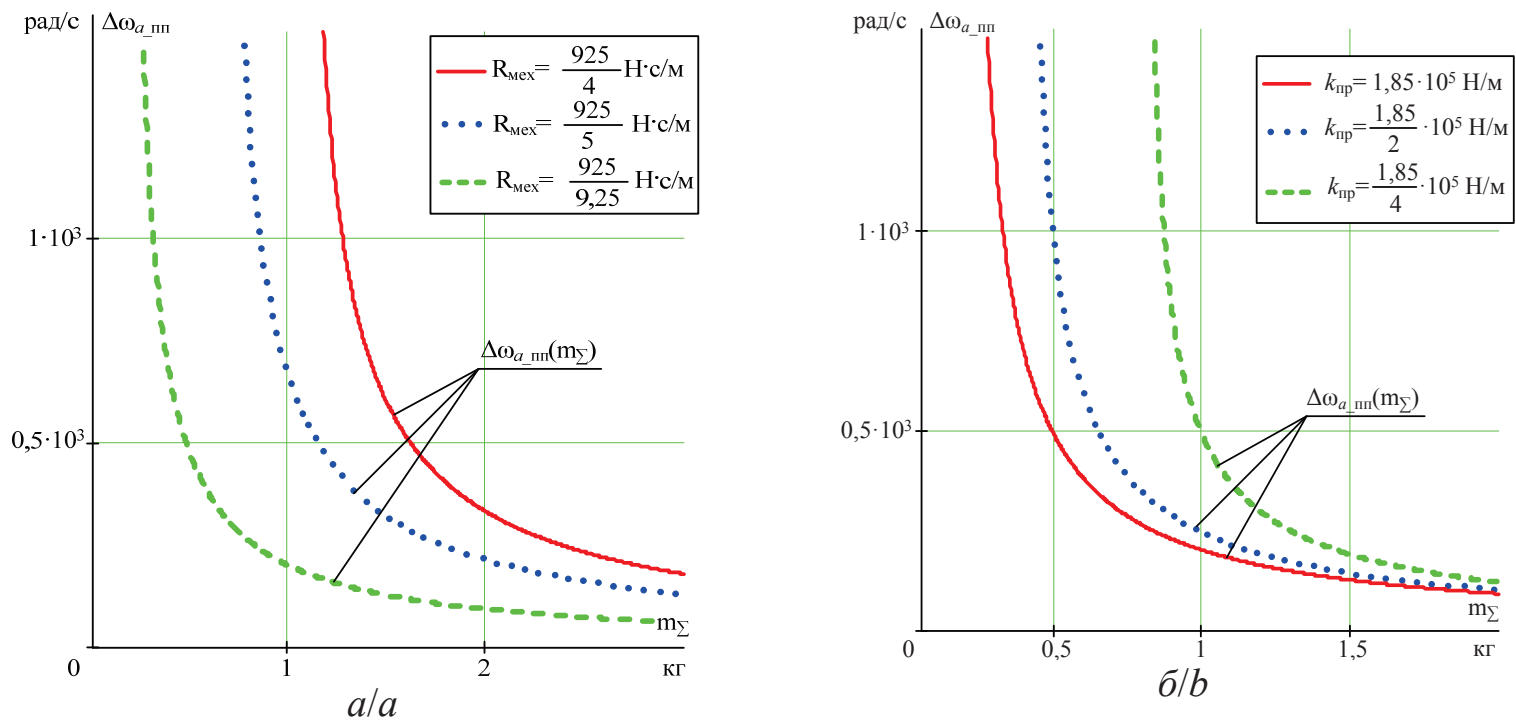

Рис. 21. Зависимость ширины полосы пропускания АЧХ ВЭМА по виброускорению от суммарной колеблющейся массы при варьировании параметров: а) коэффициента вязкого трения; б) жёсткости пружины

Fig. 21. Dependence of the bandwidth of the VEMA frequency response by vibration acceleration on the total oscillating mass with varying parameters: a) total viscous friction coefficient; $b$ ) spring stiffness

Таблииа. Результаты исследования грании применимости метода идентификации параметров механической си стемы вибрационного электромагнитного актива тора по граничным околорезонансныл частотам на основе АЧХ по виброперемещению

Table. $\quad$ Results of studying the limits of applicability of the method for identifying the parameters of vibrating elec tromagnetic activator mechanical system using near-resonance limits frequency method based on frequency res ponse of VEMA by vibration displacement

\begin{tabular}{|c|c|c|c|c|c|}
\hline$R_{\text {мех.пред }}\left(m_{\Sigma . \sigma}, k_{\text {пр.б }}\right)$ & $0,5 m_{\Sigma . \sigma}$ & $0,75 m_{\Sigma . \sigma}$ & $m_{\Sigma .6}$ & $1,25 m_{\Sigma .6}$ & $1,5 m_{\Sigma . \sigma}$ \\
\hline $0,5 k_{\text {пр.б }}$ & 267 & 327 & 377,5 & 422 & 462,5 \\
\hline $0,75 k_{\text {пр.б }}$ & 327 & 400 & 462 & 433,7 & 566 \\
\hline$k_{\text {пр.б }}$ & 377 & 462 & 534 & 597 & 654 \\
\hline $1,25 k_{\text {пр.б }}$ & 422 & 517 & 597 & 667 & 731 \\
\hline $1,5 k_{\text {пр.б }}$ & 462 & 566 & 654 & 731 & 801 \\
\hline
\end{tabular}

По данным таблицы для удобства визуализации был построен трехмерный график функции от

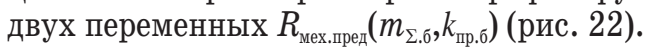

На основании рис. 22 и таблицы видно, что предельное значение коэффициента вязкого трения $R_{\text {мех.пред }}$ нелинейно возрастает при увеличении значения жесткости пружины $k_{\text {пр.б }}$ и значения суммарной присоединенной массы $m_{\Sigma .0}$. В целом метод идентификации параметров механической системы вибрационного электромагнитного активатора по граничным околорезонанстным частотам является работоспособным при слабовыраженном $\left(R_{\text {мех }}<<R_{\text {мех.пред }}\right)$ и средневыраженном $\left(R_{\text {мех }}<R_{\text {мех.пред }}\right)$ демпфировании. При условии сильного демпфирования колебаний в механическом контуре ВЭМА, 
когда $R_{\text {мех.пред }} \leq R_{\text {мех }}<R_{\text {мех.гр }}$, описанный в статье метод применять нельзя.

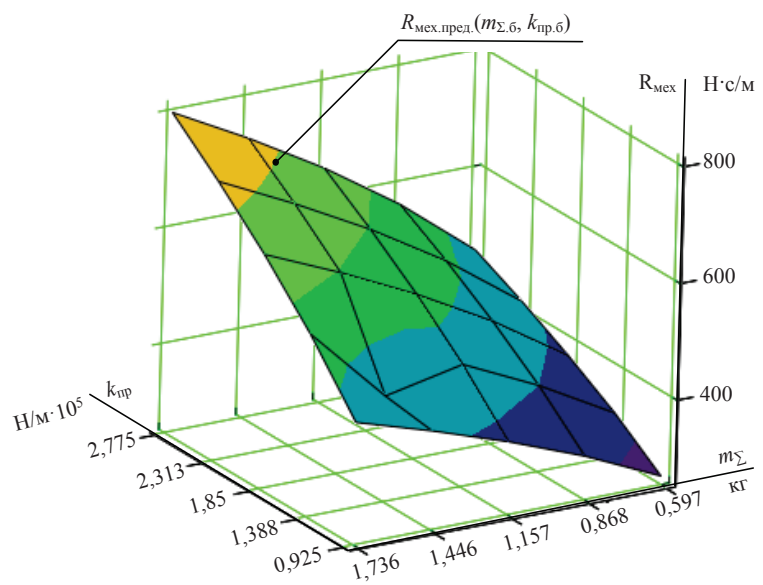

Pис. 22. Зависимость предельного значения коэффициента вязко20 трения $R_{\text {мех.пред }}$ от жесткости пружины $k_{\text {пр.б }}$ и сумлар ной присоединенной массы $m_{\Sigma, \sigma}$

Fig. 22. Dependence of the limiting value of the viscous friction coefficient $R_{\text {meh.lim }}$ on spring stiffness $k_{\mathrm{sp.b}}$ and the total oscillating mass $m_{\Sigma, b}$

\section{Заключение}

Подробно изложена уточненная версия метода идентификации параметров механической системы вибрационного электромагнитного активатора по граничным околорезонансным частотам, идея которой была ранее опубликовано в [19]. Метод по-

\section{СПИСОК ЛИТЕРАТУРЫ}

1. Neyman L.A., Neyman V.Y. Dynamic model of a vibratory electromechanical system with spring linkage // Proc. $201611^{\text {th }} \mathrm{In}-$ ternational Forum on Strategic Technology, IFOST 2016. - Novosibirsk, Russia, 2017. - № 7884234. - P. 23-27.

2. Нейман Л.А., Нейман В.Ю. Влияние механических потерь на выходные показатели электромеханической колебательной системы с электромагнитным возбуждением // Актуальные проблемы в машиностроении. - 2016. - № 3. - С. 234-240.

3. Neyman L.A., Neyman V.Y., Shabanov A.S. Vibration dynamics of an electromagnetic drive with a half-period rectifier // International Conference of Young Specialists on Micro/Nanotechnologies and Electron Devices, EDM. - Novosibirsk, Russia, 2017. № 7981805. - P. 503-506.

4. Pevchev V.P. The superexitation and efficiency relation in a short-stroke pulsed electromagnetic motor of a seismic source // Journal of Mining Science. - 2010. - V. 46. - № 6. - P. 656-665.

5. Кувшинов К.А., Мойзес Б.Б., Крауиньш П.Я. Импульсно-вибрационный источник сейсмических сигналов // Известия Томского политехнического университета. - 2010. - Т. 317. № 1. - C. 77-81.

6. Simonov B.F., Neiman V.Y., Shabanov A.S. Pulsed Linear Solenoid Actuator for Deep-Well Vibration Source // Journal of Mining Science. - 2017. - V. 53 (1). - P. 117-125.

7. Шнеерсон М.Б. Теория и практика наземной сейсморазведки. - М.: Недра, 1988. - 527 с.

8. Pevchev V.P. Science of mining machines the superexcitation and efficiency relation in a short-Stroke pulsed electromagnetic motor of a seismic source // Journal of Mining Science. - 2010. V. $46(6) .-$ P. $656-665$. зволял вычислять оценки параметров механической колебательной системы, имеющей ярко выраженный резонансный пик по граничным околорезонансным частотам. Метод имел естественное ограничение - был применим только к гармоническим колебаниям механической системы с относительно невысоким демпфированием. Описанное в статье уточнение метода заключается в новом способе составления нелинейных алгебраических уравнений для нахождения околорезонансных частот, что позволяет учесть вышеприведенные замечания.

В работе проанализированы пределы изменения коэффициента вязкого трения, при которых применение описанного метода является возможным, а корни системы алгебраических уравнений являются вещественными. Исследованы вариации предельного значения коэффициента вязкого трения, при превышении которого корни нелинейных алгебраических уравнений становятся комплексными, а применение метода становится невозможным. На амплитудно-частотных характеристиках резонансный максимум при этом становится практически сглаженным.

Разработанный метод имеет признаки универсальности и может быть рекомендован не только для идентификации параметров механической системы вибрационного электромагнитного активатора по граничным околорезонансным частотам, но и для исследования, мониторинга и настройки других механических колебательных систем, содержащих вибровозбудители.

9. Нейман Л.А., Нейман В.Ю. Моделирование динамических процессов в электромагнитных преобразователях энергии для систем генерирования силовых воздействий и низкочастотных вибраций // Известия Томского политехнического университета. Инжиниринг георесурсов. - 2015. - Т. 326. - № 4. - С. 154-162.

10. Real time prediction of suspended solids in drilling fluids. Review Article / S.C. Magalhnes, C.M. Scheid, L.A. CalHada, M.M. Lutterbach, R.S. Rezende, A.T.A. Waldmann // Journal of Natural Gas Science and Engineering. - March 2016. - V. 30. P. 164-175.

11. Данекер В.А., Рикконен С.В. Приготовление и коррекция показателей бурового раствора технологией и оборудованием BCMA // Известия Томского политехнического университета. Инжиниринг георесурсов. - 2017. - Т. 328. - № 7. - С. 86-92.

12. Pevchev V.P. Principal dimensions of the short-stroke electromagnetic motor for a seismic wave generator // Journal of Mining Science. - 2009. - V. 45. - № 4. - P. 372-381.

13. Oil recovery from tank bottom sludge using rhamnolipids / Chuhan Liu, Yin Zhang, Shanshan Sun, Lixin Huang, Li Yu, Xiaonan Liu, Ruiqiu Lai, Yijing Luo, Zhiyong Zhang, Zhongzhi Zhang // Journal of Petroleum Science and Engineering. 2018. - V. 170. - P. 14-20.

14. Analysis of oil content in drying petroleum sludge of tank bottom / Yuhua Wang, Xiaomin Zhang, Yuying Pan, Ying Chen // International Journal of Hydrogen Energy. - 2017. -V. 42. № 29. - P. 18681-18684.

15. Da S.L., Alves F.C., De França F.P. A review of the technological solutions for the treatment of oily sludges from petroleum refineries // Waste Management \& Research. - 2012. - V. 30 (10). P. 1016-1030. 
16. Lushnikov S.V., Frank Y.A., Vorobyov D.S. Oil decontamination of bottom sediments experimental work result // Earth science research journal. - 2006. - V. 10. - № 1. - P. 35-40.

17. Nekrasov V.0. Modeling bottom sediment erosion process by swirling the flow by tangential supply of oil in the tank // IOP Conference Series: Materials Science and Engineering: Transport and Storage of Hydrocarbons. - 2016. - V. 154. - 7 p. URL: http://iopscience.iop.org/article/10.1088/1757-899X/ 154/1/012003 (дата обращения 13.06.2018).

18. Burkov P.V., Burkova S.P., Samigullin V.D. Computer Simulation of Stress Strain State of Oil Gathering Pipeline Designed for Ugut Field // IOP Conference Series: Materials Science and Engineering. - 2016. - V. 125: Materials Treatment: Current Problems and Solutions. $-7 \mathrm{p}$.

19. Резонансные колебания с предельной амплитудой в вибрационом электромагнитном активаторе / А.Н. Гаврилин, Е.В. Боловин, А.С. Глазырин, С.Н. Кладиев, В.И. Полищук // Известия Томского политехнического университета. Инжиниринг георесурсов. - 2019. - Т. 330. - № 1. - С. 201-213.

20. Aver'yanov G.S., Khamitov R.N., Zubarev A.V. Dynamics of oscillatory systems with controllable shock absorbers // Russian Engineering Research. - 2008. - V. 28 (6). - P. 543-547.

21. Melkebeek J. Electrical Machines and Drives. Fundamentals and Advanced Modelling. - Cham: Springer, 2018. - 734 p.

22. Boguslawsky I., Korovkin N., Hayakawa M. Large A.C. Machines. Tokyo, Springer, 2017. $550 \mathrm{p}$.

\section{Информация об авторах}

Гаврилин A.H., кандидат технических наук, доцент отделения материаловедения Инженерной школы новых производственных технологий Национального исследовательского Томского политехнического университета.

Кладиев C.H., кандидат технических наук, доцент отделения электроэнергетики и электротехники Инженерной школы энергетики Национального исследовательского Томского политехнического университета.

Глазырин A.C., доктор технических наук, доцент отделения электроэнергетики и электротехники Инженерной школы энергетики Национального исследовательского Томского политехнического университета.

Боловин $\boldsymbol{E}$.B., ассистент отделения электроэнергетики и электротехники Инженерной школы энергетики Национального исследовательского Томского политехнического университета.

Полишук В.И., доктор технических наук, профессор кафедры электроснабжения промышленных предприятий Алтайского государственного технического университета им. И.И. Ползунова. 


\title{
IDENTIFICATION OF PARAMETERS OF VIBRATION ELECTROMAGNETIC ACTIVATOR MECHANICAL SYSTEM USING LIMITING NEAR-RESONANCE FREQUENCY
}

\author{
Alexey N. Gavrilin', \\ tom-gawral@list.ru
}

Sergey N. Kladiev', kladiev@tpu.ru

\author{
Alexander S. Glazyrin', \\ asglazyrin@tpu.ru \\ Evgeniy V. Bolovin', \\ bolovinev@mail.ru

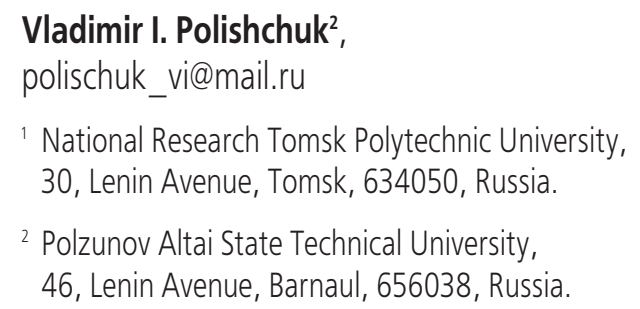

The relevance of the research is caused by the fact that vibration electromagnetic activators are effective devices for mixing suspensions, emulsions, preparing drilling fluids, liquefying highly viscous petroleum products. Armature has special design is a hydraulic rectifier. When the armature vibrates at near-resonance frequencies, deeply submerged jets are created in the treated fluid medium, which ensure high efficiency of mixing fluid medium and decrease in viscosity of petroleum products for a long time at relatively low energy consumption of the vibration electromagnetic activator. The resonant frequency of the mechanical system depends on spring stiffness, mass of the armature-activator, added mass of the fluid oscillating with the armature and viscous friction coefficient that determines the removal of energy from the vibration mechanical system. When the rheological properties of the treated fluid change, both the parameters of the mechanical vibration system and the type of frequency response of the vibration electromagnetic activator change. The method of organizing monitoring of changes in the rheological properties of a treated fluid medium with the vibration electromagnetic activator based on direct measurements, for example using viscometers, is suitable only for laboratory conditions and is not suitable for industrial implementation. According to the authors a more promising approach is based on solving an inverse mathematical problem when analyzing the vibration electromagnetic activator frequency response in particular limiting near-resonance frequency, one can get reliable estimates of the parameters of the vibration electromagnetic activator vibration mechanical system. It is convenient to use these estimates for organizing indirect monitoring of changes in the rheological properties of the treated fluid during the vibration electromagnetic activator operation and for improving the structure of the vibration electromagnetic activator automatic control system.

The main aim of the research is to design the parameters identification of the vibration electromagnetic activator mechanical system using limiting near-resonance frequency method and to determine the method's limits of applicability in high damping vibration mechanical systems.

Research methods: ordinary differential equations, Laplace transform, transmissibility, frequency response, algebraic equations. Results. The authors have obtained the analytical expressions relating the limiting near-resonance frequency to the parameters of a vibration mechanical system. Based on the latter the system of algebraic equations was obtained. The method's limits of applicability in high damping vibration mechanical systems are shown.

\section{Key words:}

Vibration electromagnetic activator, mechanical system, parameters, identification, resonance, frequency response, limiting near-resonance frequency.

\section{REFERENCES}

1. Neyman L.A., Neyman V.Y. Dynamic model of a vibratory electromechanical system with spring linkage. $11^{\text {th }}$ International Forum on Strategic Technology, IFOST 2016. Novosibirsk, Russia, 2017. No. 7884234, pp. 23-27.

2. Neyman L.A., Neyman V.Yu. Vliyaniye mekhanicheskikh poter na vykhodnye pokazateli elektromekhanicheskoy kolebatelnoy sistemy s elektromagnitnym vozbuzhdeniem [The effect of mechanical losses on the output indicators of electromechanical oscillatory system with electromagnetic excitation]. Aktualnye problemy v mashinostroyenii, 2016, no. 3, pp. 234-240.
3. Neyman L.A., Neyman V.Y., Shabanov A.S. Vibration dynamics of an electromagnetic drive with a half-period rectifier. International Conference of Young Specialists on Micro/Nanotechnologies and Electron Devices, EDM. Novosibirsk, Russia, 2017. No. 7981805, pp. 503-506.

4. Pevchev V.P. The superexitation and efficiency relation in a short-stroke pulsed electromagnetic motor of a seismic source. Journal of Mining Science, 2010, vol. 46, no. 6, pp. 656-665.

5. Kuvshinov K.A., Moizes B.B., Krauinsh P.Ya. Pulse-vibration source of seismic signals. Bulletin of the Tomsk Polytechnic University, 2010, vol. 317, no. 1, pp. 77-81. In Rus. 
6. Simonov B.F., Neiman V.Y., Shabanov A.S. Pulsed Linear Solenoid Actuator for Deep-Well Vibration Source. Journal of Mining Science, 2017, vol. 53 (1), pp. 117-125.

7. Shneerson M.B. Teoriya i praktika nazemnoy seysmorazvedki [Theory and practice of ground seismic exploration]. Moscow, $\mathrm{Ne}$ dra Publ., 1988. 527 p.

8. Pevchev V.P. Science of mining machines the superexcitation and efficiency relation in a short-Stroke pulsed electromagnetic motor of a seismic source. Journal of Mining Science, 2010, vol. 46 (6), pp. 656-665.

9. Neyman L.A., Neyman V.U. Simulation of dynamic processes in electromagnetic energy converters for force effects and lowfrequency vibrations generation systems. Bulletin of the Tomsk Polytechnic University, 2015, vol. 326, no. 4, pp. 154-162. In Rus.

10. Magalhnes S.C., Scheid C.M., CalHada L.A., Lutterbach L.M.M., Rezende R.S., Waldmann A.T.A.. Real time prediction of suspended solids in drilling fluids. Review Article. Journal of Natural Gas Science and Engineering, March 2016, vol. 30, pp. 164-175.

11. Daneker V.A., Rikkonen S.V. Preparation and correction of drilling mud by technology and equipment of vibro-jet-magnetic activation. Bulletin of the Tomsk Polytechnic University. Geo assets engineering, 2017, vol. 328, no. 7, pp. 86-92. In Rus.

12. Pevchev V.P. Principal dimensions of the short-stroke electromagnetic motor for a seismic wave generator. Journal of Mining Science, 2009, vol. 45, no. 4, pp. 372-381.

13. Chuhan Liu, Yin Zhang, Shanshan Sun, Lixin Huang, Li Yu, Xiaonan Liu, Ruiqiu Lai, Yijing Luo, Zhiyong Zhang, Zhongzhi Zhang. Oil recovery from tank bottom sludge using rhamnolipids. Journal of Petroleum Science and Engineering, 2018, vol. 170, pp. 14-20.

14. Yuhua Wang, Xiaomin Zhang, Yuying Pan, Ying Chen. Analysis of oil content in drying petroleum sludge of tank bottom. International Journal of Hydrogen Energy, 2017, vol. 42, no. 29, pp. 18681-18684.

15. Da S.L., Alves F.C., De França F.P. A review of the technological solutions for the treatment of oily sludges from petroleum refineries. Waste Management \& Research, 2012, vol. 30 (10), pp. 1016-1030.

16. Lushnikov S.V., Frank Y.A., Vorobyov D.S. Oil decontamination of bottom sediments experimental work result. Earth science research journal, 2006, vol. 10, no. 1, pp. 35-40.

17. Nekrasov V.0. Modeling bottom sediment erosion process by swirling the flow by tangential supply of oil in the tank. IOP Conference Series: Materials Science and Engineering: Transport and Storage of Hydrocarbons, 2016, vol. 154, 7 p. Available at: http://iopscience.iop.org/article/10.1088/1757-899X/ 154/1/012003 (accessed 13 June 2018).

18. Burkov P.V., Burkova S.P., Samigullin V.D. Computer Simulation of Stress Strain State of Oil Gathering Pipeline Designed for Ugut Field. IOP Conference Series: Materials Science and Engineering, 2016, vol. $125,7 \mathrm{p}$.

19. Gavrilin A.N., Bolovin E.V., Glazyrin A.S., Kladiev S.N., Polishchuk V.I. Resonant oscillations with a limiting amplitude in a vibration electromagnetic activator. Bulletin of the Tomsk Polytechnic University. Geo assets engineering, 2019, vol. 330, no. 1, pp. 201-213. In Rus.

20. Aver'yanov, G.S., Khamitov, R.N., Zubarev, A.V. Dynamics of oscillatory systems with controllable shock absorbers. Russian Engineering Research, 2008, vol. 28 (6), pp. 543-547.

21. Melkebeek J. Electrical Machines and Drives. Fundamentals and Advanced Modelling. Cham, Springer, 2018. 734 p.

22. Boguslawsky I., Korovkin N., Hayakawa M. Large A.C. Machines. Tokyo, Springer, 2017. 550 p.

23. Khamitov R.N., Aver'yanov G.S., Korchagin A.B. Pneumatic shock absorber with an active damping system. Russian Engineering Research, 2009, vol. 29 (9), pp. 871-873.

24. Binderman V.L. Teoriya mehanicheskikh kolebaniy [Theory of mechanical vibrations]. Moscow, Vysshaya Shkola Publ., 1980. $408 \mathrm{p}$.

25. Graham Kelly S. Mechanical Vibrations. Theory and applications. Boston, Cengage Learning, Inc., 2012. 898 p.

26. Glazyrin A.S., Timoshkin V.V., Tsurpal S.V., Glazyrina T.A. Identification of parameters of mechanical system on the example of a vibratory electromechanical energy converter. Bulletin of the Tomsk Polytechnic University, 2010, vol. 316, no. 4, pp. 174-177.

27. Thede L. Analog and digital filter design using C. $3^{\mathrm{d}}$ ed. New Jersey, Prentice Hall, 2005. - 352 p.

28. Linge S., Langtangen H.P. Programming for Computations MATLAB/Octav. Luxembourg, Springer Science+Business Media, 2016. 228 p.

29. Du Y., Feng W., Wang Y., Zhang G. Positive solutions for a nonlinear algebraic system with nonnegative coefficient matrix. Applied Mathematics Letters, 2017, vol. 64, pp. 150-155.

30. Di Franco P., Scarciotti G., Astolfi A. A note on the stability of nonlinear differential-algebraic systems. IFAC-PapersOnLine, 2017, vol. 50, Iss. 1, pp. 7421-7426.

Received: 11 February 2019.

\section{Information about the authors}

Alexey N. Gavrilin, Cand. Sc., associate professor, National Research Tomsk Polytechnic University.

Sergey N. Kladiev, Cand. Sc., associate professor, National Research Tomsk Polytechnic University.

Alexander S. Glazyrin, Dr. Sc., associate professor, National Research Tomsk Polytechnic University.

Evgeniy V. Bolovin, assistant, National Research Tomsk Polytechnic University.

Vladimir I. Polishchuk, Dr. Sc., professor, Polzunov Altai State Technical University. 